\title{
Theoretical Design and Characteristics Analysis of a Quasi-Zero Stiffness Isolator Using a Disk Spring as Negative Stiffness Element
}

\author{
Lingshuai Meng, Jinggong Sun, and Wenjuan Wu \\ Institute of Medical Equipment, Academy of Military Medical Sciences, 106 Wandong Road, Hedong District, Tianjin 300161, China \\ Correspondence should be addressed to Jinggong Sun; sunjg@vip.sina.com
}

Received 10 August 2014; Revised 27 December 2014; Accepted 29 December 2014

Academic Editor: Kumar V. Singh

Copyright (C) 2015 Lingshuai Meng et al. This is an open access article distributed under the Creative Commons Attribution License, which permits unrestricted use, distribution, and reproduction in any medium, provided the original work is properly cited.

\begin{abstract}
This paper presents a novel quasi-zero stiffness (QZS) isolator designed by combining a disk spring with a vertical linear spring. The static characteristics of the disk spring and the QZS isolator are investigated. The optimal combination of the configurative parameters is derived to achieve a wide displacement range around the equilibrium position in which the stiffness has a low value and changes slightly. By considering the overloaded or underloaded conditions, the dynamic equations are established for both force and displacement excitations. The frequency response curves (FRCs) are obtained by using the harmonic balance method (HBM) and confirmed by the numerical simulation. The stability of the steady-state solution is analyzed by applying Floquet theory. The force, absolute displacement, and acceleration transmissibility are defined to evaluate the isolation performance. Effects of the offset displacement, excitation amplitude, and damping ratio on the QZS isolator and the equivalent system (ELS) are studied. The results demonstrate that the QZS isolator for overloaded or underloaded can exhibit different stiffness characteristics with changing excitation amplitude. If loaded with an appropriate mass, excited by not too large amplitude, and owned a larger damper, the QZS isolator can possess better isolation performance than its ELS in low frequency range.
\end{abstract}

\section{Introduction}

The requirements for low-frequency isolators arise in many scientific and industrial fields, ranging from the isolation of precision instruments for gravitational wave detections to the design of seat suspension systems for motors $[1,2]$. For traditional passive linear isolators, a smaller stiffness is desired to achieve a smaller natural frequency so that it can attenuate low-frequency vibrations [3]. In this case, a larger static deflection is unavoidable in practical applications. To overcome the limitation between isolation performance and static deflection, passive nonlinear isolators have been used to obtain a high static stiffness resulting in a small static deflection and a low dynamic stiffness resulting in a small natural frequency [4]. By choosing the appropriate configurative and geometric parameters of nonlinear isolators, a quasi-zero stiffness (QZS) isolator possessing zero dynamic stiffness at the static equilibrium position can be realized [5].
There are a number of ways to design a QZS isolator by combining a negative stiffness element with a positive element. Ibrahim [6] presented a comprehensive assessment of recent advances in nonlinear isolators with good ultralow frequency isolation performance. Alabuzhev et al. [7] covered the fundamental theory and many prototypes of vibration isolation systems characterized with QZS. Peng et al. [8] used six rods and a tension spring to achieve the QZS property. Platus [9] utilized two compressed bars hinged at the center to be the negative element. Zhang et al. [10] added a beam under axial force to a positive stiffness spring. Carrella et al. [11] proposed a high-static-low-dynamic stiffness (HSLDS) isolator with a vertical linear spring in parallel with two oblique linear springs. Kovacic et al. [12] conducted further research by using two nonlinear prestressed oblique nonlinear springs as negative element. Le and Ahn [13] studied a low-frequency isolator for vehicle seat theoretically and experimentally, in 


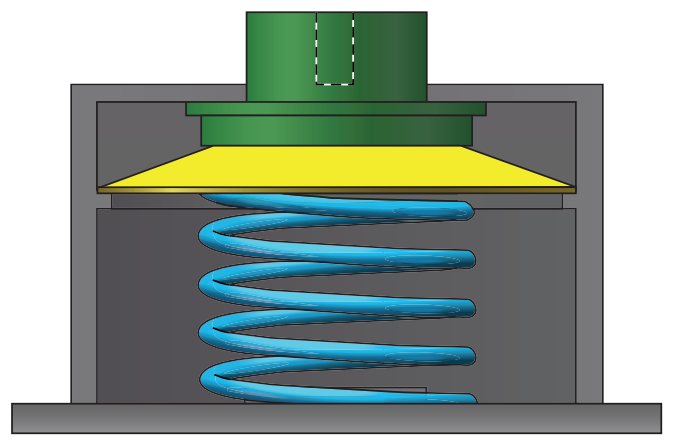

FIGURE 1: Prototype model of the proposed QZS isolator.

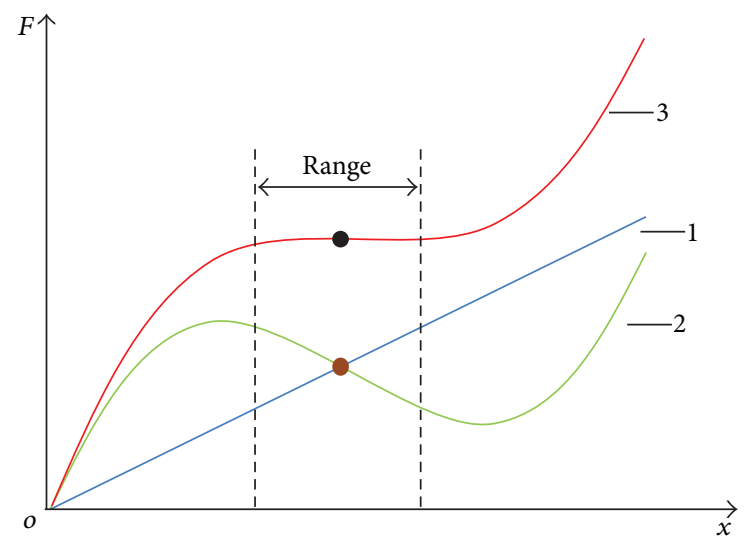

FIGURE 2: The principle for achieving the QZS property.

which the negative stiffness element is configured by a horizontal spring in series with a bar. Liu et al. [14] studied the characteristics of a QZS isolator using Euler buckled beam as negative stiffness corrector. Magnetic springs were also introduced to QZS isolators. Carrella et al. [15] proposed a model with two linear springs and three permanent magnets arranged in an attracting configuration. Robertson et al. [16] examined another isolator that exhibits localized zero stiffness by the interaction between a floating magnet and two fixed permanent magnets. Zhou and Liu [17] developed a HDLDS isolator comprising a mechanical spring and an electromagnet. Xu et al. [18] investigated a QZS isolator based on a prototype of combining an appropriate vertical spring with two pitched bars connected with magnets.

In this paper, we propose a new QZS isolator by combining a disk spring with a vertical linear spring as shown in Figure 1. Compared with other negative stiffness elements, taking a disk spring as negative stiffness element can offer greater support capacity because the disk spring can bear great load with small deflection and supply a certain restoring force at the flatten state. Therefore, the new QZS isolator is suitable for being used in the occasion with space limitation for isolators. Meanwhile, its axial nonlinear restoring force enables the isolator to achieve the QZS property at the static equilibrium position. The principle for achieving the QZS property is presented in Figure 2. Curve 1 is the typical forcedisplacement curve for the vertical linear spring, curve 2 is

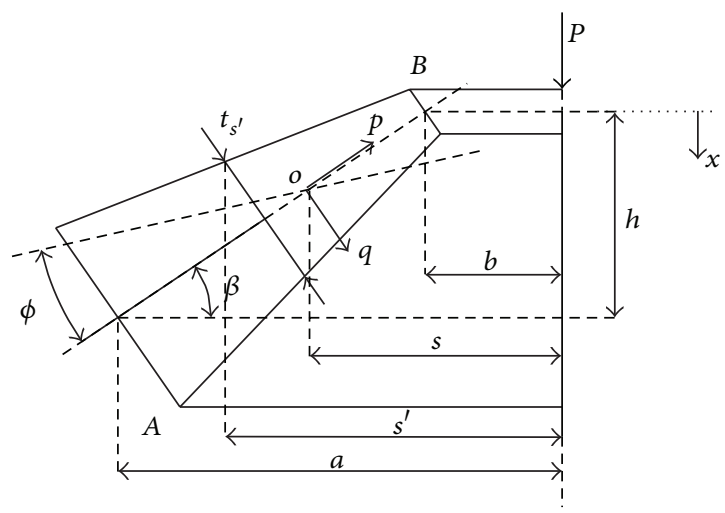

FIgURE 3: The disk spring under axial force.

the typical force-displacement curve for the disk spring, and curve 3 is the combined curve. In the displacement range around the static equilibrium position, the negative stiffness offered by the disk spring offsets the positive stiffness offered by the linear spring, thus leading to a QZS isolator. And the restoring force of the disk spring at the static equilibrium position is positive, which means the isolator can own greater support capacity.

The aims of this paper are to design configurative parameters and investigate the characteristics and effects of overload or underload on the isolation performance of the designed QZS isolator. The paper is organized as follows. In Section 2, the static characteristics of the disk spring as well as the QZS isolator are presented. In addition, the optimization of the QZS isolator is conducted to get a wider displacement range around the static equilibrium position in which the stiffness is lower and changes slightly. The dynamic modeling and solution are illustrated in Section 3, followed by the numerical simulations and the stability analysis in Section 4. In Section 5, the frequency response curves (FRCs) and transmissibilities for the force and displacement excitations are studied by considering the overload or underload condition. This section also discusses the effects of the damping ratio on the QZS isolator. Finally, some conclusions on the performance of the QZS isolator are drawn in Section 6.

\section{Static Characteristics of the QZS Isolator}

2.1. Disk Spring. A disk spring of variable thickness loaded axially is shown in Figure 3, where $a$ is the external radius, $b$ is the internal radius, $s$ is the distance of neutral axis to center, $h$ is the free height, $t(p)$ is the thickness at $p$ position, $\beta$ is the initial cone angle of disk, and $\phi$ is the change of cone angle due to load $P$. The law of variable thickness is defined by

$$
t(p)=T_{0}+T_{1} p,
$$

where $T_{0}=t_{s^{\prime}}(1+\kappa((a+b) /(a-b))-2(\kappa s /(a-b)))$ and $T_{1}=\left(2 t_{s^{\prime}} /(a-b)\right) \kappa \cdot t_{s^{\prime}}$ is the thickness at radius $s^{\prime}=(a+b) / 2$, and $\kappa=\left(t_{b}-t_{a}\right) / 2 t_{s^{\prime}}$ is a nondimensional parameter which defines the form of the spring shown in Figure 4.

Starting from the Almen and Laszlo theory [19] and following the indications of la Rosa et al. [20], the relationship 


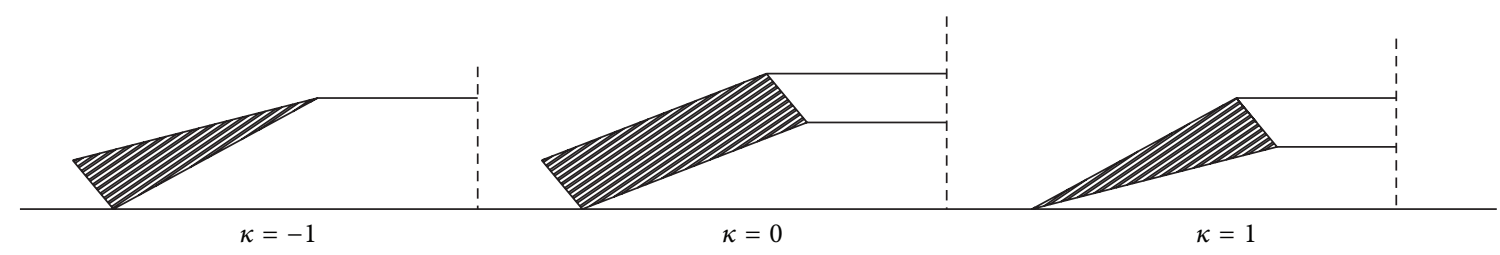

FIGURE 4: Disk spring with thickness varying along the generatrix of the cone.

between the applied axial force $P$ and axial deflection $x$ can be derived:

$$
P=\frac{2 \pi E x}{(a-b)^{2}\left(1-\mu^{2}\right)}\left[(h-x)\left(h-\frac{x}{2}\right) M+N\right],
$$

where $x=(a-b) \phi, E$ is Young's modulus of the disk spring, and $\mu$ is Poisson's ratio. The parameters $M$ and $N$ are defined as

$$
\begin{gathered}
M=\frac{1}{(a-b)^{2}}\left[T_{0} U_{1}+T_{1} U_{2}\right], \\
N=\frac{1}{12}\left[T_{0}^{3} U_{3}+3 T_{0}^{2} T_{1} U_{4}+3 T_{0} T_{1}^{2} U_{1}+T_{1}^{3} U_{2}\right],
\end{gathered}
$$

in which

$$
\begin{aligned}
U_{1} & =\frac{1}{2}\left(a^{2}-b^{2}\right)-2 s(a-b)+s^{2} \ln \frac{a}{b}, \\
U_{2} & =-\frac{1}{3}\left(a^{3}-b^{3}\right)+\frac{3}{2} s\left(a^{2}-b^{2}\right)-3 s^{2}(a-b)+s^{3} \ln \frac{a}{b}, \\
U_{3} & =\ln \frac{a}{b} \\
U_{4} & =-(a-b)+s \ln \frac{a}{b}, \\
s & =\frac{(a-b)^{2}}{-2 \kappa(a-b)+(a-b) \ln (a / b)+\kappa(a+b) \ln (a / b)} .
\end{aligned}
$$

With the disk spring moving down from the initial position $x=0$, it starts to provide a restoring force $F_{d}$. When the disk spring is in a horizontal line which means $x=h$, the deflection and restoring force can be expressed by the point $\left(x_{e}, F_{d e}\right)$. Note that the force-displacement curve is symmetric about the point as $x$ changes during the range $[0,2 h]$. Set this point as the origin of a new vertical displacement coordinate $u$. The relationship between $u$ and $x$ is $u=x-h$. It is convenient to define the following nondimensional parameters:

$$
\begin{aligned}
\widehat{u} & =\frac{u}{a}, \\
\widehat{h} & =\frac{h}{a}, \\
\widehat{M} & =\frac{M}{a},
\end{aligned}
$$

$$
\begin{gathered}
\widehat{N}=\frac{N}{a^{3}}, \\
\widehat{\Gamma}=\frac{1}{(1-\widehat{b})^{2}}, \\
\widehat{F}_{d}=\frac{F_{d}}{2 \pi E a^{2} /\left(1-\mu^{2}\right)} .
\end{gathered}
$$

The relationship of nondimensional force-displacement for the disk spring can be derived as

$$
\widehat{F}_{d}=\widehat{\Gamma}\left[\frac{\widehat{M}}{2} \widehat{u}^{3}+\left(\widehat{N}-\frac{\widehat{M}}{2} \widehat{h}^{2}\right) \widehat{u}+\widehat{N} \widehat{h}\right],
$$

where $\widehat{F}_{d}$ is the nondimensional restoring force and $\widehat{u}$ is the nondimensional displacement.

By differentiating (6) with respect to the nondimensional displacement $\widehat{u}$, the nondimensional stiffness of the disk spring $\widehat{k}_{d}$ can be expressed by

$$
\widehat{k}_{d}=\widehat{\Gamma}\left(\frac{3 \widehat{M}}{2} \widehat{u}^{2}+\widehat{N}-\frac{\widehat{M}}{2} \widehat{h}^{2}\right) .
$$

Considering the parameters $\widehat{M}>0$ and $\widehat{\Gamma}>0$, it can be seen obviously that the stiffness of the disk spring is symmetric about $\widehat{u}=\widehat{u}_{e}=0$ and reaches the minimum value $\widehat{k}_{d \text { min }}$ at this position. The disk spring owns continuous negative stiffness region when the parameters meet the condition $\widehat{h}>\sqrt{2 \widehat{N} / \widehat{M}}$. Then the minimum negative value of the nondimensional stiffness and the continuous negative stiffness region can be derived as follows:

$$
\begin{aligned}
& \widehat{k}_{d \min }=\widehat{\Gamma}\left(\widehat{N}-\frac{\widehat{M}}{2} \widehat{h}^{2}\right), \\
& \widehat{u} \in\left(-\sqrt{\frac{\widehat{h}^{2}}{3}-\frac{2 \widehat{N}}{3 \widehat{M}}}, \sqrt{\frac{\widehat{h}^{2}}{3}-\frac{2 \widehat{N}}{3 \widehat{M}}}\right) .
\end{aligned}
$$

For the disk springs with the same internal and external radius, and the same thickness $t_{s^{\prime}}$, the nondimensional stiffness-displacement characteristics of different parameter $\kappa$ are presented in Figure 5 according to (7). The displacement region where the nondimensional stiffness is negative is also indicated. The disk springs of variable thickness possess larger negative stiffness region than that of constant thickness 


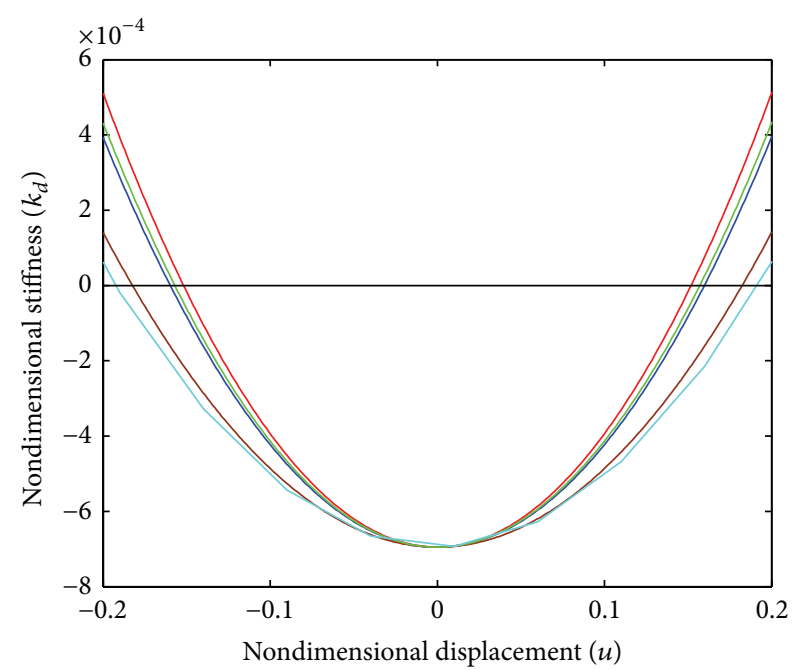

Figure 5: Nondimensional stiffness characteristics of the disk springs for different $|\kappa|$. "Red line" $\kappa=0$ (constant thickness); "blue line" $\kappa=0.5$; "brown line" $\kappa=1$; "green line" $\kappa=-0.5$; "cyan line" $\kappa=-1$.

when they get the same minimum negative value of the nondimensional stiffness. Meanwhile, it can be seen that the larger the value $|\kappa|$, the larger the negative stiffness region.

2.2. The QZS Isolator. The disk spring of negative stiffness can be connected parallelly with a positive stiffness spring to achieve a low equivalent stiffness. The schematic of the proposed QZS isolator is shown in Figure 6. A vertical linear spring $k_{v}$ and a viscous damper $c$ are in parallel with a disk spring acting as the negative stiffness element. At the initial position, there are no deformations both for the vertical linear spring and the disk spring. Here the weight of the isolated mass is ignored. As displayed in Figure 6(a), when the mass moves downward and has a certain displacement $x$, it sustains two vertical forces including an axial restoring force from the disk spring and a restoring force from the vertical linear spring. Thus, the vertical restoring force of the isolator $F_{r}$ can be derived:

$$
F_{r}=k_{v} x+\frac{2 \pi E x}{(a-b)^{2}\left(1-\mu^{2}\right)}\left[(h-x)\left(h-\frac{x}{2}\right) M+N\right] .
$$

By substituting $u=x-h$ into (9) and introducing the nondimensional restoring force $\widehat{F}_{r}=F_{r} /\left(k_{v} a\right)$, the nondimensional restoring force can be expressed by

$$
\widehat{F}_{r}=\widehat{u}+\widehat{h}+\lambda \widehat{\Gamma}\left[\frac{\widehat{M}}{2} \widehat{u}^{3}+\left(\widehat{N}-\frac{\widehat{M}}{2} \widehat{h}^{2}\right) \widehat{u}+\widehat{N} \widehat{h}\right]
$$

where $\lambda=2 \pi E a /\left[k_{v}\left(1-\mu^{2}\right)\right]$ is defined as the stiffness ratio between the disk spring and the vertical linear spring and the other parameters have the same meaning with that in 2.1 . Differentiating (10) with respect to the nondimensional displacement $\widehat{u}$, one can get the nondimensional stiffness of the isolator

$$
\widehat{k}=1+\lambda \widehat{\Gamma}\left(\frac{3 \widehat{M}}{2} \widehat{u}^{2}+\widehat{N}-\frac{\widehat{M}}{2} \widehat{h}^{2}\right),
$$

where $\widehat{k}=k / k_{v}$ is the nondimensional stiffness.

When the disk spring is horizontal, that is, $\widehat{u}=\widehat{u}_{e}=0$, the nondimensional stiffness of the isolator is symmetric about the position and has the minimum value. It is found that the larger the defined stiffness ratio, the smaller the nondimensional stiffness of the isolator. However, the nondimensional stiffness of the isolator should not be negative, otherwise the isolator will be unstable. By substituting $\widehat{u}=\widehat{u}_{e}=$ 0 into (11) and referring to the condition that the disk spring has negative stiffness in Section 2.1, one can get that the configurative parameters of the isolator must satisfy the condition:

$$
\sqrt{\frac{2 \widehat{N}}{\widehat{M}}}<\widehat{h} \leq \sqrt{\frac{2}{\widehat{M}}\left(\frac{1}{\lambda \widehat{\Gamma}}+\widehat{N}\right) .}
$$

In operation, the isolator is prospected to reach static equilibrium at the position $\widehat{u}=\widehat{u}_{e}=0$ and has zero stiffness after being loaded with an appropriate mass. By setting (11) to zero at the static equilibrium position, the desired stiffness ratio can be derived by

$$
\lambda_{\mathrm{QZS}}=\frac{2}{\widehat{\Gamma}\left(\widehat{M} \widehat{h}^{2}-2 \widehat{N}\right)} .
$$

2.3. Optimization of the QZS Isolator. Except for owning the QZS property, it is desirable for the isolator to have a wide range of nondimensional displacement $\widehat{d}$ from the static equilibrium position in which the nondimensional stiffness gets a low value. Substituting $\widehat{u}=\widehat{u}_{e} \pm \widehat{d}= \pm \widehat{d}$ into (11), one can get relationship between the nondimensional displacement $\widehat{d}$ and the configurative parameters:

$$
\widehat{d}=\sqrt{\frac{2}{3 \widehat{M}}\left(\frac{\widehat{k}-1}{\lambda \widehat{\Gamma}}+\frac{\widehat{M}}{2} \widehat{h}^{2}-\widehat{N}\right)} .
$$

According to the practical engineering conditions [21] and the analysis above, the configurative parameters $\widehat{b}, \widehat{t}_{s^{\prime}}, \widehat{h}$, and $\kappa$ are chosen from the range $0.1 \leq \widehat{b} \leq 0.8,0.01 \leq \widehat{t}_{s^{\prime}} \leq$ $0.1, \sqrt{2 \widehat{N} / \widehat{M}}<\widehat{h} \leq \sqrt{2(1 / \lambda \widehat{\Gamma} \widehat{M}+\widehat{N} / \widehat{M})}$, and $-1 \leq \kappa \leq$ 1 . Among all the combinations of these configurative parameters and the defined stiffness ratio $\lambda$ calculated using (13), only those for which the defined stiffness ratio $\lambda$ is positive are considered. The 50CrVA is used to be the material of the disk spring here. Its Young's modulus is $E=206 \mathrm{GPa}$, Poisson's ratio is $\mu=0.3$, and ultimate stress is $S=1275 \mathrm{MPa}$. It is worthy of note that the optimization criteria include the achievement of the largest displacement from the static equilibrium position, at which the non-dimensional stiffness is equal to that of the vertical linear spring alone, that is, $\widehat{k}=1$, the condition that the nondimensional stiffness cannot be 


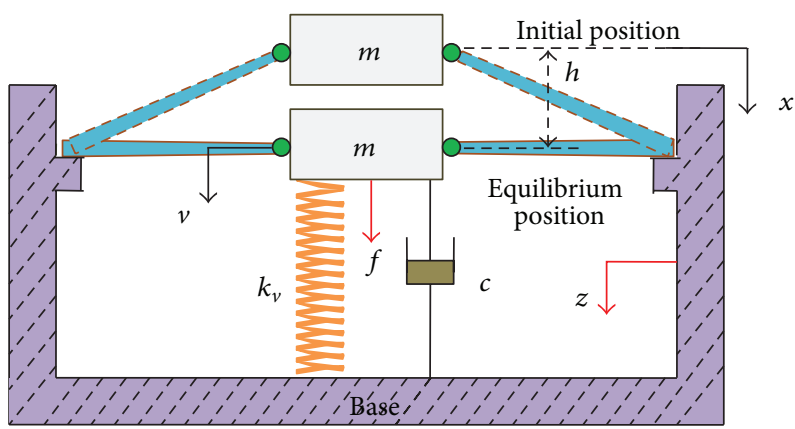

(a) Ideal isolator with load balanced at the zero stiffness equilibrium position

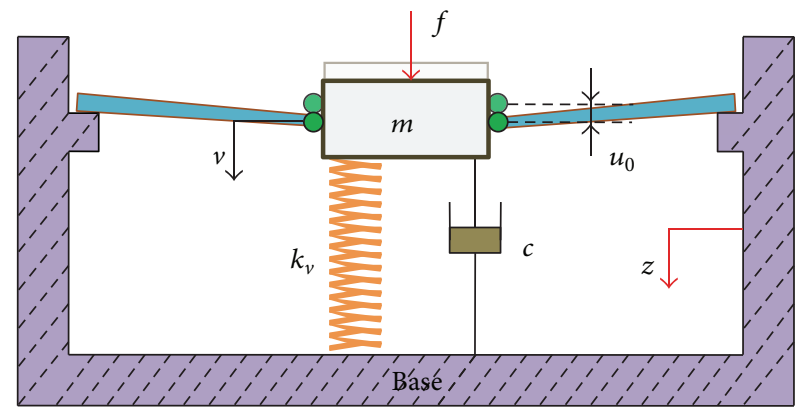

(b) Disturbed isolator for overloaded

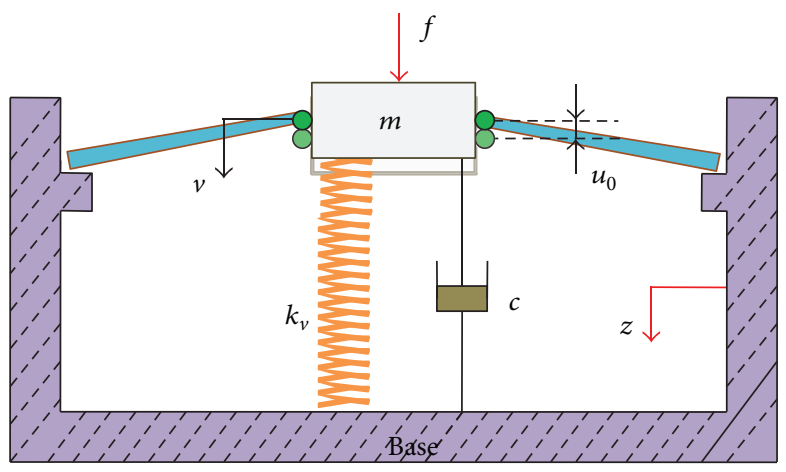

(c) Disturbed isolator for underloaded

FIGURE 6: Schematic representation of the proposed QZS isolator.

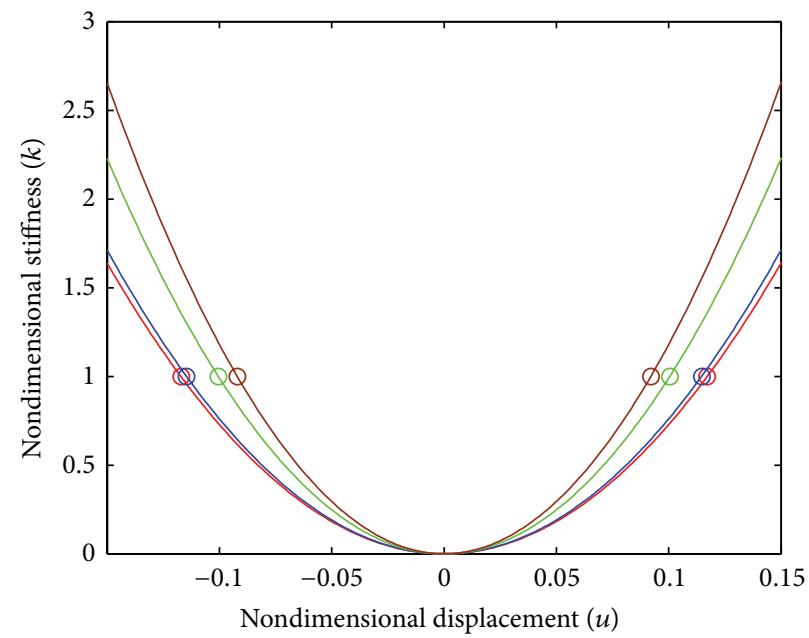

Figure 7: Nondimensional stiffness characteristics of the QZS isolator for different configurative parameters. "Red line" curve 1; "blue line" curve 2; "green line" curve 3; "brown line" curve 4.

negative, and the requirement that the nondimensional stiffness changes slightly with the tolerance of $\Delta \widehat{k}=0.2$ for $\Delta \widehat{u}=0.01$ during the neighborhood of the static equilibrium position. In addition, the maximum stress of the disk spring occurs at the lower outer edge $A$ or the upper inner edge $B$. Moreover, only those disk springs for which the maximum stress cannot be larger than the ultimate stress are taken into account [20].
The optimal result is the combination of $\widehat{b}=0.12, \widehat{t}_{s^{\prime}}=$ $0.1, \widehat{h}=0.2447$, and $\kappa=-0.16$. As shown in Figure 7 , the nondimensional stiffness-displacement curve for the optimal configurative parameters (Curve 1) is plotted. The other three curves for combinations of the configurative parameters satisfying the optimization criteria listed in Table 1 are also plotted for comparison. The circles denote the largest displacement calculated using (14) when $\widehat{k}=1$. It is 
TABLE 1: The configurative parameters of the QZS isolator satisfying the optimization criteria.

\begin{tabular}{lcccc}
\hline Curve & $\widehat{b}$ & $\widehat{t}_{s^{\prime}}$ & $\widehat{h}$ & $\kappa$ \\
\hline 1 & 0.12 & 0.1 & 0.2447 & -0.16 \\
2 & 0.1 & 0.1 & 0.2402 & -0.19 \\
3 & 0.1 & 0.1 & 0.2192 & -0.24 \\
4 & 0.1 & 0.09 & 0.1995 & -0.24 \\
\hline
\end{tabular}

worth noting that the optimal QZS isolator possesses a very small stiffness in the neighborhood of the static equilibrium position and a smaller stiffness for larger displacements from the equilibrium position.

\section{Dynamic Modeling and Solution}

Combining (10) and (13), the nondimensional restoring force of the QZS isolator can be derived as

$$
\widehat{F}_{r}=\eta+\chi \widehat{u}^{3},
$$

where $\eta=\widehat{M} \widehat{h}^{3} /\left(\widehat{M} \widehat{h}^{2}-2 \widehat{N}\right)$ and $\chi=\widehat{M} /\left(\widehat{M} \widehat{h}^{2}-2 \widehat{N}\right)$.

As shown in Figure 6(a), the ideal isolator loaded with an appropriate mass can keep balance at the static equilibrium position $\widehat{u}=\widehat{u}_{e}=0$. And the static equilibrium position is a zero stiffness position. However, the isolator is more likely to balance at $\widehat{u}=+\widehat{u}_{0}$ for overloaded or $\widehat{u}=-\widehat{u}_{0}$ for underloaded in the practical applications. As shown in Figures 6(b) and 6(c) separately, the disturbed isolators for overloaded and underloaded have an offset displacement $\widehat{u}_{0}$ from the static equilibrium position. Their static equations can be given by

$$
k_{v} a\left(\eta \pm \chi \widehat{u}_{0}^{3}\right)=m g .
$$

The nondimensional force-displacement and stiffnessdisplacement curves of the disturbed isolators for overloaded and underloaded are plotted in Figure 8. The new static equilibrium positions are denoted by “*” and "o" separately while the zero stiffness position is denoted by "•." It is worthy of note that the effects of the overload and underload on the isolator cannot be ignored because the new equilibrium positions have an offset displacement $\widehat{u}_{0}$ from the zero stiffness position and the nondimensional stiffness of new equilibrium positions would not be zero. According to Figure 6, two types of excitations are considered: one is the harmonic force excitation $f(t)=F \cos (\omega t)$ on the mass; the other one is the harmonic displacement excitation $z(t)=Z \cos (\omega t)$ on the base. By using Newton's second law of motion, one can achieve the dynamic equations separately for the two types of excitations given above:

$$
\begin{aligned}
& m \ddot{\delta}+c \dot{\delta}+k_{v} a\left[\eta+\chi\left(\widehat{\delta} \pm \widehat{u}_{0}\right)^{3}\right]-m g=F \cos (\omega t), \\
& m \ddot{y}+c \dot{y}+k_{v} a\left[\eta+\chi\left(\widehat{y} \pm \widehat{u}_{0}\right)^{3}\right]-m g=m \omega^{2} Z \cos (\omega t),
\end{aligned}
$$

where $\delta$ is the displacement from the new equilibrium position and $y=\delta-z$ is the relative displacement between
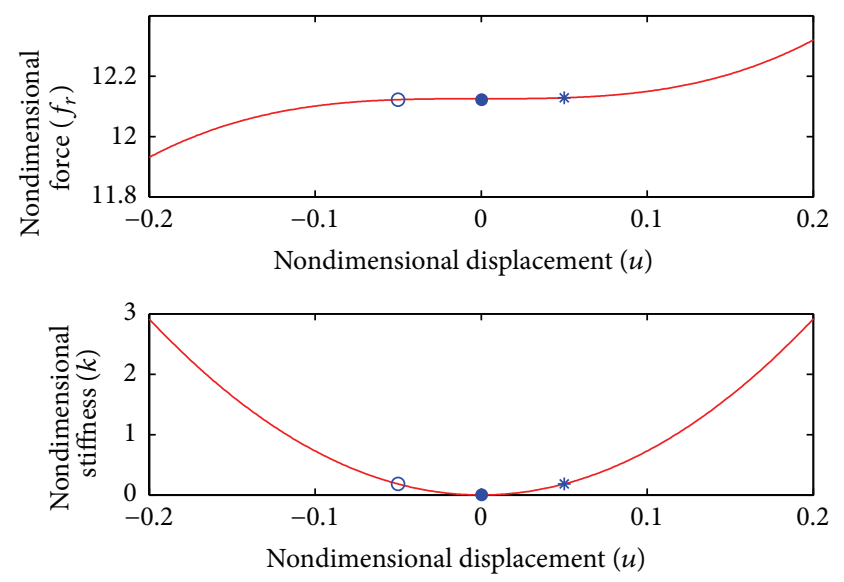

Figure 8: Nondimensional force-displacement and stiffnessdisplacement curves with the optimal configurative parameters. “" zero stiffness position shown in Figure 6(a); “*” equilibrium position in Figure 6(b); “o” equilibrium in Figure 6(c).

the base and the mass. Combining (16) and introducing the nondimensional parameters as follows:

$$
\begin{gathered}
\omega_{n}=\sqrt{\frac{k_{v}}{m},} \\
\tau=\omega_{n} t, \\
\Omega=\frac{\omega}{\omega_{n}}, \\
\xi=\frac{c}{2 m \omega_{n}}, \\
\widehat{F}=\frac{F}{m \omega_{n}^{2} D}, \\
\widehat{Z}=\frac{Z}{D},
\end{gathered}
$$

(17a) and (17b) can be rewritten as the nondimensional form:

$$
\begin{aligned}
& \widehat{\delta}^{\prime \prime}+2 \xi \widehat{\delta}^{\prime}+3 \chi \widehat{u}_{0}^{2} \widehat{\delta} \pm 3 \chi \widehat{u}_{0} \widehat{\delta}^{2}+\chi \widehat{\delta}^{3}=\widehat{F} \cos (\Omega \tau), \\
& \widehat{y}^{\prime \prime}+2 \xi \widehat{y}^{\prime}+3 \chi \widehat{u}_{0}^{2} \widehat{y} \pm 3 \chi \widehat{u}_{0} \widehat{y}^{2}+\chi \widehat{y}^{3}=\Omega^{2} \widehat{Z} \cos (\Omega \tau) .
\end{aligned}
$$

Equations (19a) and (19b) can be expressed by a uniform dynamic equation for simplicity:

$$
\widehat{v}^{\prime \prime}+2 \xi \widehat{v}^{\prime}+\alpha_{1} \widehat{v} \pm \alpha_{2} \widehat{v}^{2}+\chi \widehat{v}^{3}=\gamma \rho \cos (\Omega \tau),
$$

where $\alpha_{1}=3 \chi \widehat{u}_{0}^{2}, \alpha_{2}=3 \chi \widehat{u}_{0}, \rho$ is the amplitude of the harmonic excitations, and $\gamma=1$ for the force excitation while $\gamma=$ $\Omega^{2}$ for the displacement excitation. Known as the HelmholtzDuffing equation, (20) can be recast in the form of a Duffing oscillator under asymmetric excitation. Applying the transformation $\widehat{e}=\widehat{v} \pm \alpha_{2} /(3 \chi)=\widehat{v} \pm \widehat{u}_{0}$ [22], (20) can be rewritten as

$$
\widehat{e}^{\prime \prime}+2 \xi \hat{e}^{\prime}+\chi \widehat{e}^{3}= \pm \alpha+\gamma \rho \cos (\Omega \tau),
$$


where $\alpha=\chi \widehat{u}_{0}^{3}$. Considering only the primary resonance response, the harmonic balance method (HBM) can be employed to get the approximate steady-state solution of (21). The steady-state solution is assumed to be

$$
\widehat{e}=A_{0}+A_{1} \cos (\Omega \tau+\varphi) .
$$

By substituting (22) into (21) and equating constant terms, the coefficients of the terms containing $\cos (\Omega \tau)$ and $\sin (\Omega \tau)$ separately to zero, one can get the steady-state solution expressed by the following algebraic equations in terms of a bias term $A_{0}$, the amplitude of the harmonic term $A_{1}$, and the phase $\varphi$ :

$$
\begin{gathered}
\chi A_{0}^{3}+\frac{3}{2} \chi A_{0} A_{1}^{2}= \pm \alpha, \\
-\Omega^{2} A_{1}+3 \chi A_{0}^{2} A_{1}+\frac{3}{4} \chi A_{1}^{3}=\gamma \rho \cos (\varphi), \\
-2 \xi \Omega A_{1}=\gamma \rho \sin (\varphi) .
\end{gathered}
$$

Combining (23a)-(23c), the implicit equation for the amplitude of the bias term $A_{0}$ is

$$
\begin{aligned}
& 25 \chi^{3} A_{0}^{9}-20 \chi^{2} \Omega^{2} A_{0}^{7}-15 \chi^{2} \alpha A_{0}^{6} \\
& \quad+4 \chi \Omega^{2}\left(\Omega^{2}+4 \xi^{2}\right) A_{0}^{5}+16 \chi \alpha \Omega^{2} A_{0}^{4} \\
& \quad+3 \chi\left(2 \gamma^{2} \rho^{2}-3 \alpha^{2}\right) A_{0}^{3}-4 \alpha \Omega^{2}\left(\Omega^{2}+4 \xi^{2}\right) A_{0}^{2} \\
& \quad+4 \alpha^{2} \Omega^{2} A_{0}-\alpha^{3}=0 .
\end{aligned}
$$

By solving (24), which is also a quadratic polynomial about $\Omega^{2}$, the implicit equation for the peak amplitude of the bias term for the force and displacement excitations, that is, $A_{0 p}^{f}$ and $A_{0 p}^{d}$, can be derived separately as

$$
\begin{aligned}
-20 \chi^{3} \xi^{2} A_{0 p}^{f 9}+8 \chi^{2} \xi^{4} A_{0 p}^{f 7}+36 \chi^{2} \alpha \xi^{2} A_{0 p}^{f 6} \\
-3 \chi^{2} \rho^{2} A_{0 p}^{f 5}-16 \chi \alpha \xi^{4} A_{0 p}^{f 4} \\
\quad-12 \chi \alpha^{2} \xi^{2} A_{0 p}^{f 3}+3 \chi \alpha \rho^{2} A_{0 p}^{f 2} \\
\quad+8 \alpha^{2} \xi^{4} A_{0 p}^{f}-4 \alpha^{3} \xi^{2}=0, \\
\left(-75 \chi^{4} \rho^{2}-80 \chi^{3} \xi^{2}\right) A_{0 p}^{d 9}+32 \chi^{2} \xi^{4} A_{0 p}^{d 7} \\
+\left(45 \chi^{3} \alpha \rho^{2}+144 \chi^{2} \alpha \xi^{2}\right) A_{0 p}^{d 6}-64 \chi \alpha \xi^{4} A_{0 p}^{d 4} \\
+\left(27 \chi^{2} \alpha^{2} \rho^{2}-48 \chi \alpha^{2} \xi^{2}\right) A_{0 p}^{d 3} \\
+32 \alpha^{2} \xi^{4} A_{0 p}^{d}+\left(3 \chi \alpha^{3} \rho^{2}-16 \alpha^{3} \xi^{2}\right)=0 .
\end{aligned}
$$

The frequencies corresponding to the peak responses $\Omega_{p}^{f}$ and $\Omega_{p}^{d}$ for the two types of excitations can be obtained separately:

$$
\begin{aligned}
& \Omega_{p}^{f}=\sqrt{\frac{5 \chi^{2} A_{0 p}^{f 6}-4 \chi \xi^{2} A_{0 p}^{f 4}-4 \chi \alpha A_{0 p}^{f 3}+4 \alpha \xi^{2} A_{0 p}^{f}-\alpha^{2}}{2 \chi A_{0 p}^{f 4}-2 \alpha A_{0 p}^{f}}}, \\
& \Omega_{p}^{d}=\sqrt{\frac{5 \chi^{2} A_{0 p}^{d 6}-4 \chi \xi^{2} A_{0 p}^{d 4}-4 \chi \alpha A_{0 p}^{d 3}+4 \alpha \xi^{2} A_{0 p}^{d}-\alpha^{2}}{2 \chi A_{0 p}^{d 4}+3 \chi \rho^{2} A_{0 p}^{d 2}-2 \alpha A_{0 p}^{d}}} .
\end{aligned}
$$

And the amplitude of the harmonic term $A_{1}$ and its peak amplitudes $A_{1 p}^{f}$ and $A_{1 p}^{d}$ for the two types of excitations can be derived using (23a). Note that (24)-(26b) are only valid for the disturbed isolator for overloaded with an equilibrium position at $\widehat{u}=+\widehat{u}_{0}$. For the disturbed isolator for underloaded with an equilibrium position at $\widehat{u}=-\widehat{u}_{0}$, the steadystate solution can be achieved by transforming $\widehat{u}_{0}$ to $-\widehat{u}_{0}$ in (24).

For the ideal isolator with an equilibrium position at $\widehat{u}=$ 0 , the steady-state solutions for the two types of excitations can be obtained by setting $\widehat{u}_{0}=0$ and following the procedure above. Its dynamic equation is

$$
\widehat{v}^{\prime \prime}+2 \xi \widehat{v}^{\prime}+\chi \widehat{v}^{3}=\gamma \rho \cos (\Omega \tau) .
$$

Then the implicit amplitude frequency equation can be derived as

$$
\frac{9}{16} \chi^{2} A_{1}^{6}-\frac{3}{2} \chi \Omega^{2} A_{1}^{4}+\Omega^{2}\left(\Omega^{2}+4 \xi^{2}\right) A_{1}^{2}-\gamma^{2} \beta^{2}=0 .
$$

The peak amplitude of the responses $A_{1 p}^{f}$ and $A_{1 p}^{d}$ and the frequencies corresponding to the peak responses $\Omega_{p}^{f}$ and $\Omega_{p}^{d}$ for the two types of excitations can be achieved as follows:

$$
\begin{gathered}
A_{1 p}^{f}=\sqrt{\frac{2 \xi^{3}+\sqrt{4 \xi^{6}+3 \chi \rho^{2}}}{3 \chi \xi}}, \\
A_{1 p}^{d}=\frac{8 \xi^{2}}{\sqrt{48 \chi \xi^{2}-9 \chi^{2} \rho^{2}}}, \\
\Omega_{p}^{f}=\sqrt{\frac{\sqrt{4 \xi^{6}+3 \chi \rho^{2}}-6 \xi^{3}}{4 \xi}}, \\
=\sqrt{\frac{(9 / 2) \chi \rho^{2} \xi^{6}-12 \xi^{8}}{12 \xi^{6}-(45 / 4) \chi \rho^{2} \xi^{4}+(27 / 8) \chi^{2} \rho^{4} \xi^{2}-(81 / 256) \chi^{3} \rho^{6}}} .
\end{gathered}
$$




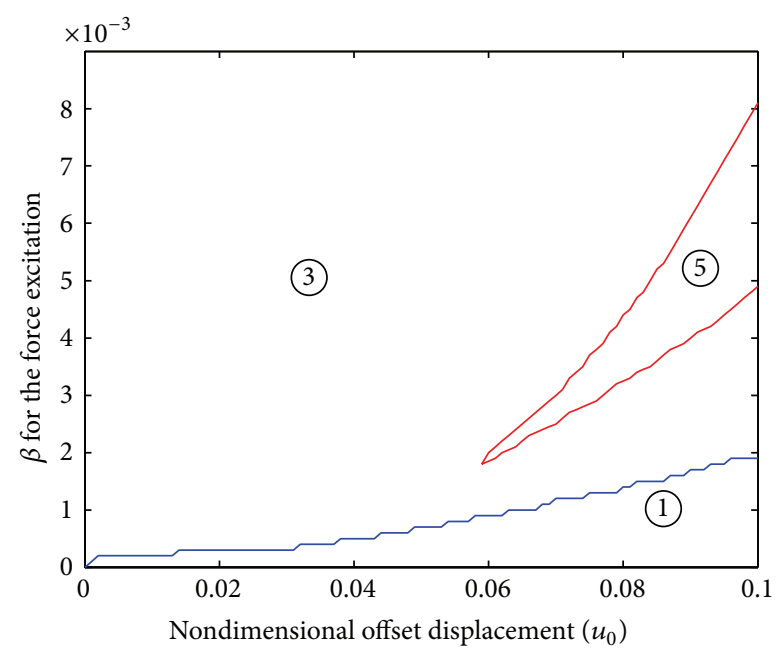

(a) For the force excitation

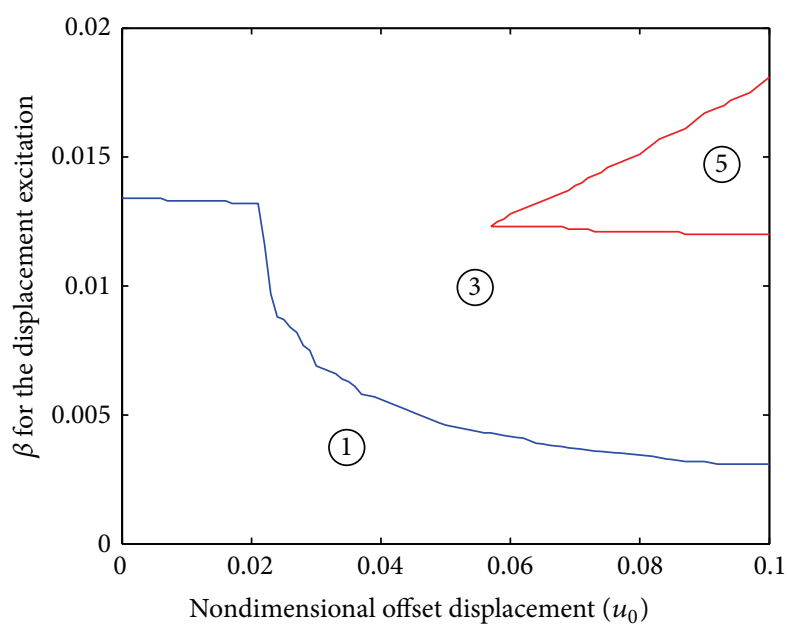

(b) For the displacement excitation

FIGURE 9: The maximum number of the steady-state solutions: one, three, or five, as a function of the offset displacement $\widehat{u}_{0}$ and the excitation amplitude $\rho$ for the optimal configurative parameters and $\xi=0.03$.

By removing the disk spring, the equivalent linear system (ELS) to the QZS isolator can be obtained. The dynamic equation of the ELS is

$$
\widehat{v}^{\prime \prime}+2 \xi \widehat{v}^{\prime}+\widehat{v}=\gamma \rho \cos (\Omega \tau)
$$

The amplitude of the steady-state response for (30) is

$$
A_{1}=\frac{\gamma \rho}{\sqrt{\left(1-\Omega^{2}\right)^{2}+(2 \xi \Omega)^{2}}} .
$$

\section{Numerical Simulations and Stability}

4.1. Numerical Simulations and Confirmations. According to the analysis of Kovacic et al. [23], the system under the asymmetric excitation may have a maximum number of one, three, or five steady-state values and exhibit multiple jumps for different combinations of excitation amplitudes. The optimal configurative parameters illuminated in Section 2.3 and the damping ratio $\xi=0.03$ are used to conduct the following investigations. Then the value of the constant term $\alpha$ in (21) is only related to the offset displacement $\widehat{u}_{0}$. Based on Descartes' rule of signs [24] and the analysis above, the ways in which the maximum number of the steady-state values of $A_{0}$ and $A_{1}$ depend on the offset displacement $\widehat{u}_{0}$ and the excitation amplitude $\rho$ are shown in Figure 9. It is worthy of note that there are one steady-state value for the small excitation amplitude while three and five steady-state values are the major phenomenon.

Due to the occurrence of three and five steady-state values, the phenomenon of multiple jumps may appear. To illustrate these cases, the FRCs with a maximum number of one, three, and five for the force excitation corresponding to the combinations: $\widehat{u}_{0}=0.04, \rho=3 \times 10^{-4} ; \widehat{u}_{0}=0.04, \rho=$ $1.2 \times 10^{-3} ; \widehat{u}_{0}=0.07, \rho=2.7 \times 10^{-3}$, respectively, and that for the displacement excitation corresponding to the combinations: $\widehat{u}_{0}=0.04, \rho=3 \times 10^{-3} ; \widehat{u}_{0}=0.04, \rho=$ $1.3 \times 10^{-2} ; \widehat{u}_{0}=0.07, \rho=1.3 \times 10^{-2}$, respectively are plotted in Figure 10. Note that the steady-state solution is evaluated by considering only the component of the response at the excitation frequency. It is necessary to make the numerical simulation to confirm the accuracy of the appropriate solutions obtained by the HBM. By applying the MATLAB ode45 function, the exact solutions are achieved and plotted in Figure 10 using the symbols "*" and "o." "*” and "o" denote the exact solutions obtained separately by the increasing and decreasing frequency, respectively. It is found that both the bias term $A_{0}$ and the amplitude of the harmonic term $A_{1}$ are calculated reasonably well in the frequency range using the HBM.

As shown in Figures 10(b) and 10(e), the bias term $A_{0}$ and the amplitude of the harmonic term $A_{1}$ follow the route marked by 1-2-3-4-5-6 as the frequency increases. Point 2 is a jump-down point for $A_{0}$ and a jump-up point for $A_{1}$, while point 4 is a jump-up point for $A_{0}$ and a jump-down point for $A_{1}$. If the frequency decreases, the route is 6-7-8-9-10. Point 7 is a jump-down point for $A_{0}$ and a jump-up point for $A_{1}$, while point 9 is a jump-up point for $A_{0}$ and a jump-down point for $A_{1}$. For the both two types of excitations, the bias term $A_{0}$ and the amplitude of the harmonic term $A_{1}$ can occur a jump-down and a jump-up phenomenon. Different from Figures 10(b) and 10(e), it is worthy of note that the route shown in Figures 10(c) and 10(f) is changed to 1-2-3-6 as the frequency increases. For the two types of excitations, there is only one jump point for the bias term $A_{0}$ and the amplitude of the harmonic term $A_{1}$ such that point 2 is a jump-down point for $A_{0}$ and a jump-up point for $A_{1}$.

4.2. Stability of the Steady-State Solution. On account of the appearance of multiple values for the steady-state solution, the stability of the steady-state solution should be 

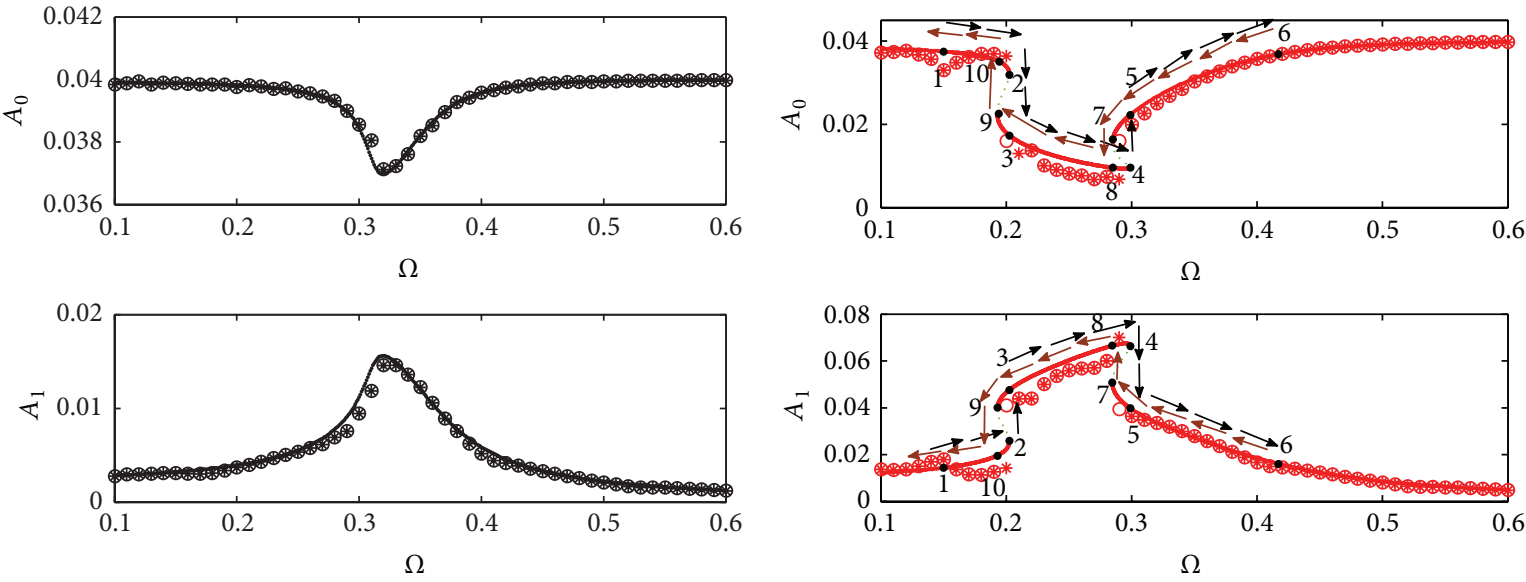

(a) $\widehat{u}_{0}=0.04, \rho=3 \times 10^{-4}$

(b) $\widehat{u}_{0}=0.04, \rho=1.2 \times 10^{-3}$
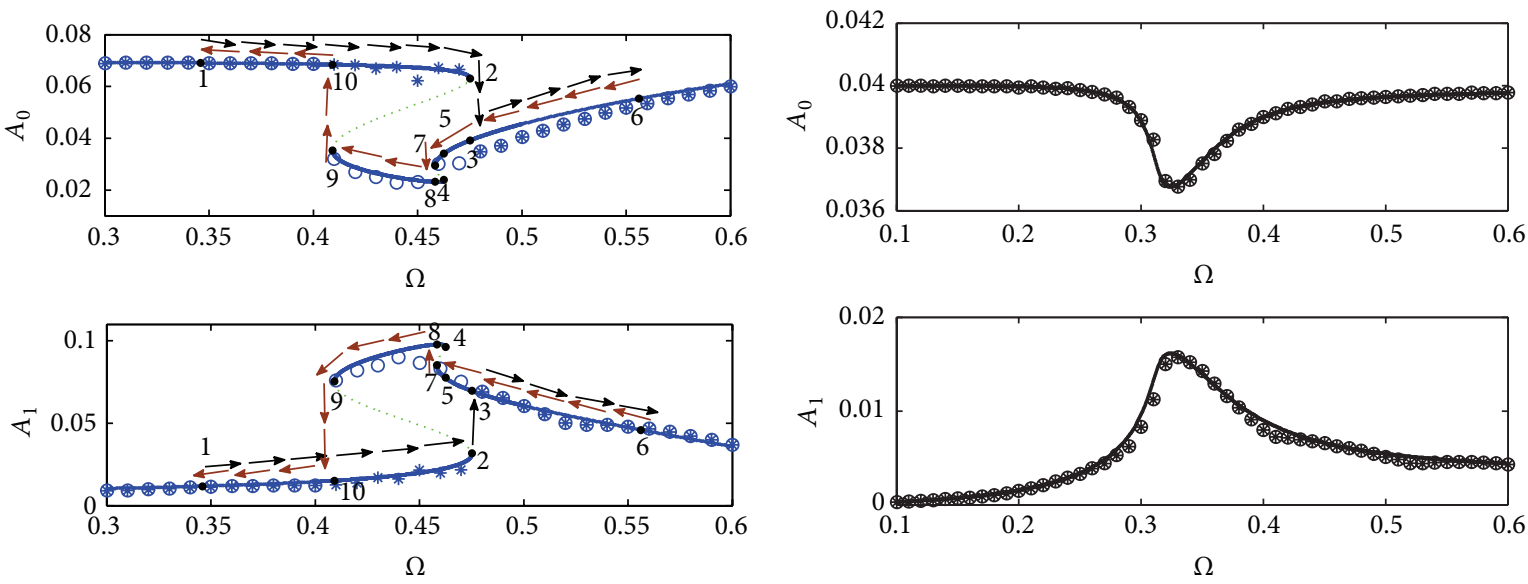

(c) $\widehat{u}_{0}=0.07, \rho=2.7 \times 10^{-3}$

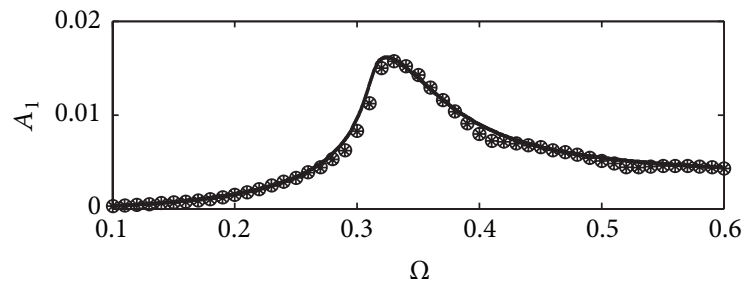

(d) $\widehat{u}_{0}=0.04, \rho=3 \times 10^{-3}$
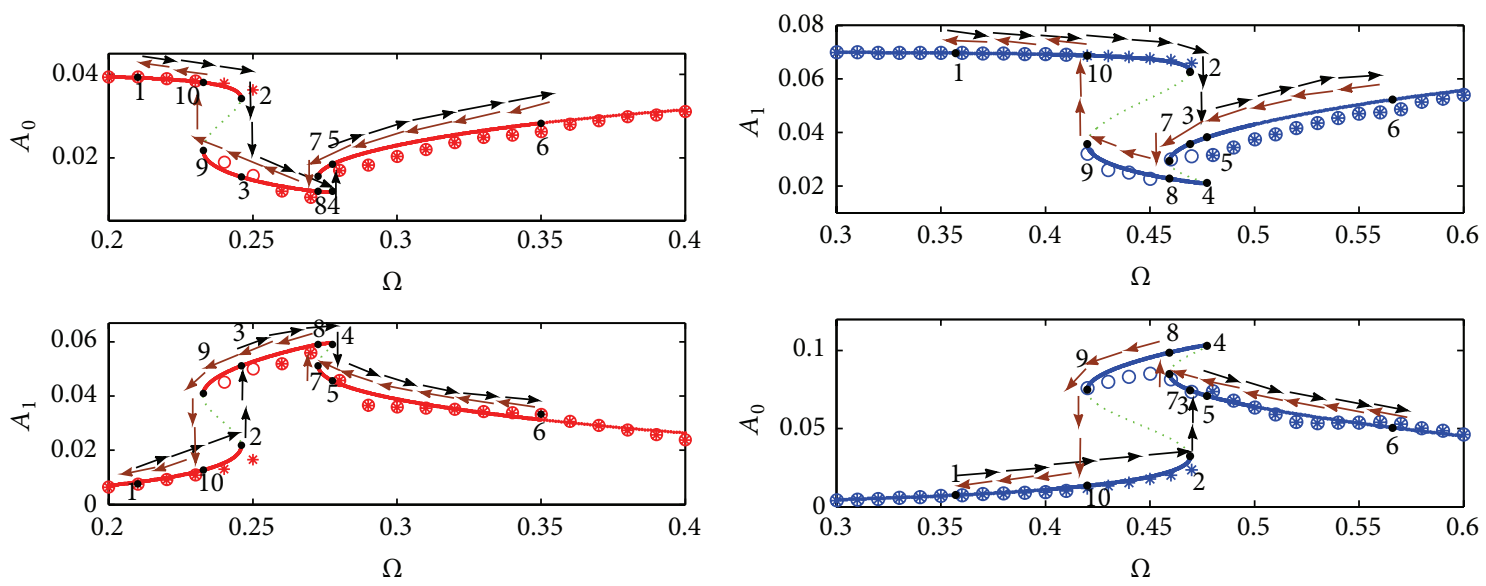

(e) $\widehat{u}_{0}=0.04, \rho=1.3 \times 10^{-2}$

(f) $\widehat{u}_{0}=0.07, \rho=1.3 \times 10^{-2}$

FIgURE 10: FRCs of the bias term $A_{0}$ and the amplitude of the harmonic term $A_{1}$ for the optimal configurative parameters and $\xi=0.03$. $((\mathrm{a})-(\mathrm{c}))$ The force excitation; ((d)-(f)) the displacement excitation; "green dotted line" unstable solution; "*" numerical solution obtained by increasing frequency; "o" numerical solution obtained by decreasing frequency. 
TABLE 2: The offset displacement and excitation amplitude for the two types of excitations.

\begin{tabular}{lcc}
\hline $\begin{array}{l}\text { Type of the } \\
\text { excitation }\end{array}$ & $\widehat{u}_{0}$ & Parameters \\
\hline Force & $0,0.04,0.07$ & $\begin{array}{c}3 \times 10^{-4}, 6 \times 10^{-4}, 1.2 \times 10^{-3}, \\
2.7 \times 10^{-3}, 2 \times 10^{-2}\end{array}$ \\
& & $3 \times 10^{-3}, 8 \times 10^{-3}, 1.3 \times 10^{-2}$, \\
Displacement & $0,0.04,0.07$ & $2 \times 10^{-2}, 1.5 \times 10^{-1}$ \\
\hline
\end{tabular}

investigated. Superposing a small nondimensional perturbation $\widehat{\varepsilon}(\tau)$ on (22), one can get

$$
\widehat{e}=A_{0}+A_{1} \cos (\Omega \tau+\varphi)+\widehat{\varepsilon}(\tau) .
$$

By substituting (32) into (21) and combining the steadystate solution equation (22), the equation for the perturbation can be derived as

$$
\widehat{\varepsilon}^{\prime \prime}+2 \xi \widehat{\varepsilon}^{\prime}+3 \chi\left[A_{0}+A_{1} \cos (\Omega \tau+\varphi)\right]^{2} \widehat{\varepsilon}=0 .
$$

$$
\left[\begin{array}{ccc}
\psi^{2}+\iota_{0} & \frac{\iota_{1}}{2} \cos (\varphi) & -\frac{\iota_{1}}{2} \sin (\varphi) \\
-\sin (\varphi) & -2 \psi \Omega-\frac{\iota_{2}}{2} \sin (2 \varphi) & \psi^{2}-\Omega^{2}+\iota_{0}-\frac{\iota_{2}}{2} \cos (2 \varphi) \\
\cos (\varphi) & \psi^{2}-\Omega^{2}+\iota_{0}+\frac{\iota_{2}}{2} \cos (2 \varphi) & 2 \psi \Omega-\frac{\iota_{2}}{2} \sin (2 \varphi)
\end{array}\right] \times\left\{\begin{array}{c}
C \\
\sin (\theta) \\
\cos (\theta)
\end{array}\right\}=0
$$

Nontrivial solutions exist if the determinant of the matrix in (36) vanishes, which can be derived as

$$
\iota_{0} \iota_{2}^{2}-\iota_{1}^{2} \iota_{2}+2 \iota_{1}^{2}\left(\iota_{0}-\Omega^{2}\right)-4 \iota_{0}\left(\iota_{0}-\Omega^{2}\right)^{2}=0 .
$$

Equation (37) is the boundary between the stable and unstable regions and the unstable region can be determined by

$$
\iota_{0} \iota_{2}^{2}-\iota_{1}^{2} \iota_{2}+2 \iota_{1}^{2}\left(\iota_{0}-\Omega^{2}\right)-4 \iota_{0}\left(\iota_{0}-\Omega^{2}\right)^{2}<0
$$

As shown in Figure 10, the green dotted lines of the FRCs represent the unstable regions. If there are three steady-state solutions occurring at the same frequency, two of them are stable and one unstable for the two types of excitations shown in Figures 10(b) and 10(e). When five steady-state solutions occur as shown in Figures 10(c) and 10(f), three of them are stable and two unstable.

\section{Dynamic Characteristics of the QZS Isolator}

For the two types of excitations, the values of the offset displacement $\widehat{u}_{0}$ and excitation amplitude $\rho$ listed in Table 2 are chosen to study their effects on the QZS isolator. The optimal configurative parameters and $\xi=0.03$ are applied for the QZS isolator. And the excitation amplitude $\rho$ and damping ratio $\xi$ of the ELS are chosen as same as that of the QZS isolator for comparison convenience.
Supposing that $\widehat{\varepsilon}(\tau)=e^{-\varsigma \tau} \widehat{\sigma}(\tau),(33)$ is changed to be the form of Hill's equation:

$$
\widehat{\sigma}^{\prime \prime}+3 \chi\left[\iota_{0}+\iota_{1} \cos (\Omega \tau+\varphi)+\iota_{2} \cos 2(\Omega \tau+\varphi)\right] \widehat{\sigma}=0,
$$

where $\iota_{0}=3 \chi A_{0}^{2}+(3 / 2) A_{1}^{2}-\xi^{2}, \iota_{1}=6 \chi A_{0} A_{1}, \iota_{2}=(3 / 2) \chi A_{1}^{2}$. Under parametric excitations, the system modeled by (34) can exhibit resonance whenever the excitation frequency is equal to $2 \sqrt{l_{0}} / n$, in which $\sqrt{\iota_{0}}$ is the normalized frequency of the system and $n$ is an integer [25]. It is worthy of note that the second unstable region is of interest in the stability analysis, that is, $n=2$, because the steady-state solution obtained by HBM owns the same frequency with the excitation frequency. According to Floquet theory, the solution of (34) can be assumed to be

$$
\widehat{\sigma}=e^{\psi \tau}(C+\sin (\Omega \tau+\theta)),
$$

where $\psi$ is the characteristic Floquet exponent. By inserting (35) into (34) and applying the HBM, one can achieve that

5.1. Effects of the Offset Displacement and Excitation Amplitude on the FRCs. The FRCs of the QZS isolator and the ELS are illustrated in Figure 11 for the force excitation. By observing Figure 11, one can conclude that a decrease in the offset displacement $\widehat{u}_{0}$ results in a decrease in the bias term $A_{0}$ and resonance frequency $\Omega_{p}^{f}$ (the excitation frequency corresponding to the peak response) and an increase in the peak amplitude of the harmonic term $A_{1 p}^{f}$ when the excitation amplitude is fixed. It is worthy of note that the bias term disappears for the ideal isolator with zero offset displacement. For the disturbed isolator with a nonzero offset displacement, the amplitude of the harmonic term $A_{1}$ increases as the excitation amplitude increases. But its peak amplitude of the harmonic term $A_{1 p}^{f}$ cannot be larger than that of the ideal isolator. And the effect of the excitation amplitude on the bias term $A_{0}$ is only obvious around the resonance frequency, in which larger excitation amplitude leads to smaller peak amplitude of the bias term $A_{0 p}^{f}$. When the frequency is far away from the resonance frequency, the bias term $A_{0}$ changes little and approaches the value of the offset displacement $\widehat{u}_{0}$. It is also worth noting that the resonance frequency of the disturbed isolator decreases at first, increases later, and becomes larger than that of the ELS finally as the excitation amplitude increases. However, both the amplitude of the response $A_{1 p}^{f}$ and resonance frequency $\Omega_{p}^{f}$ of the ideal isolator increase 

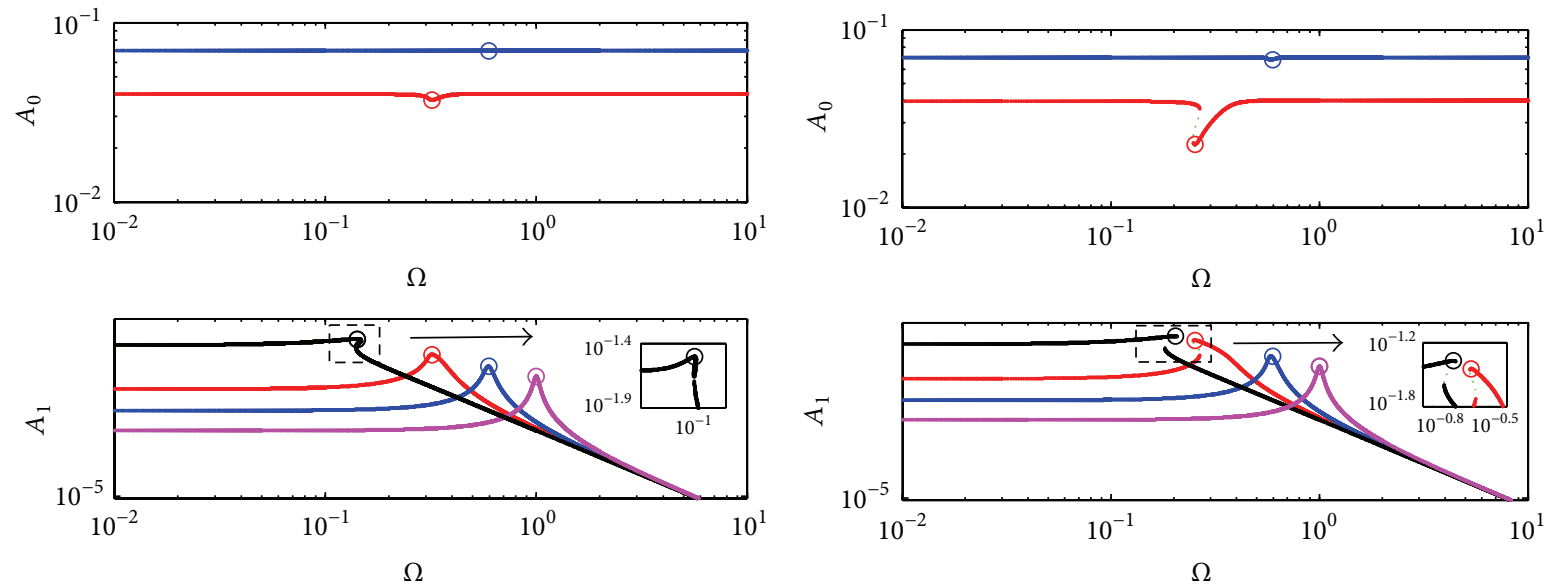

(a) $\rho=3 \times 10^{-4}$

(b) $\rho=6 \times 10^{-4}$
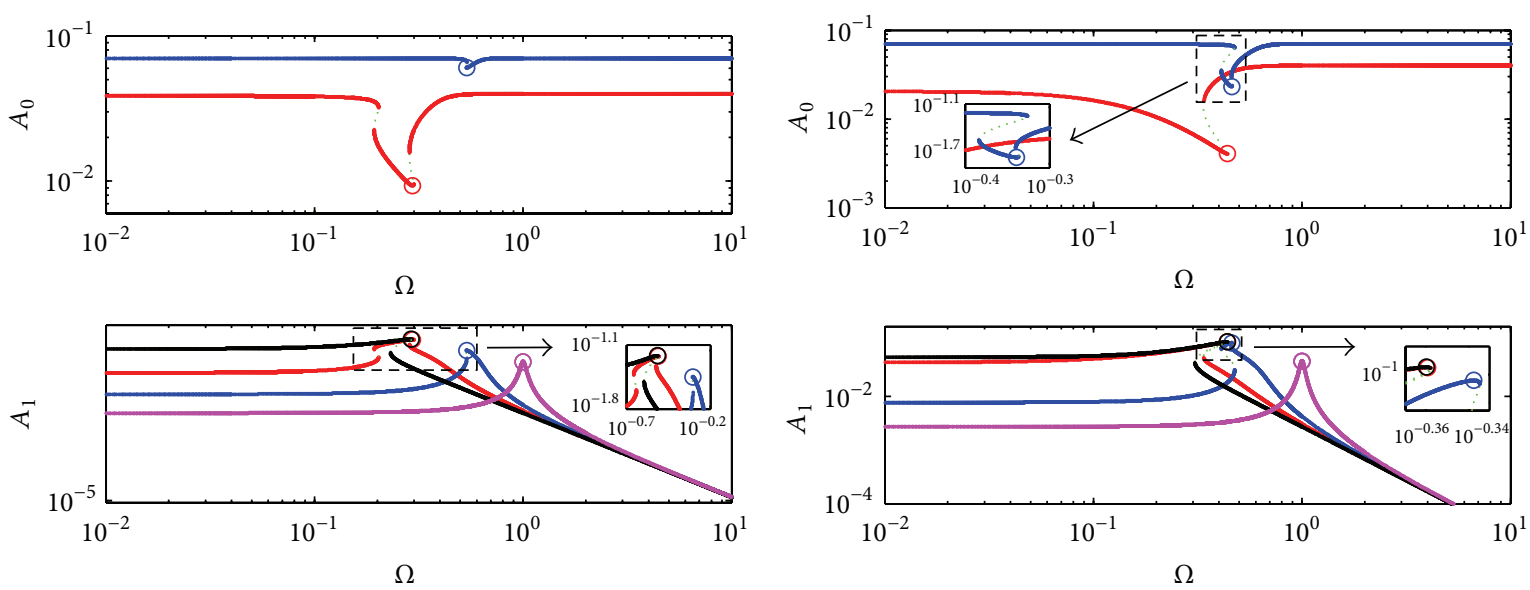

(c) $\rho=1.2 \times 10^{-3}$

(d) $\rho=2.7 \times 10^{-3}$
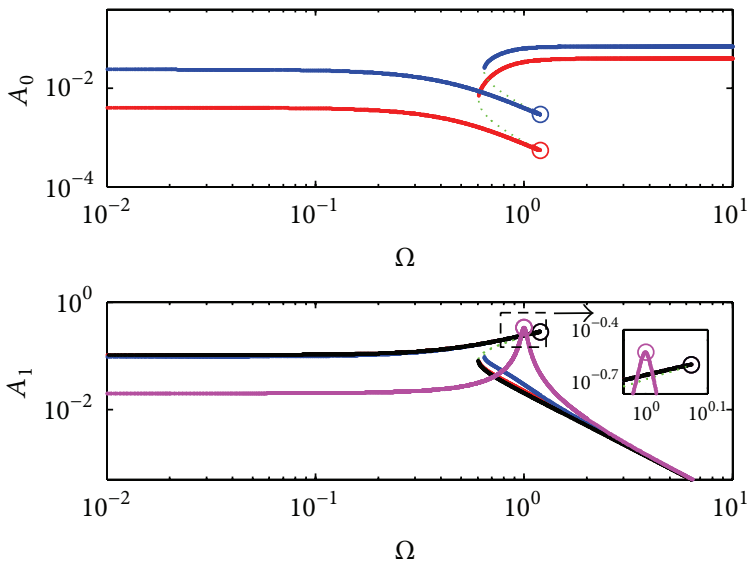

(e) $\rho=2 \times 10^{-2}$

FIGURE 11: FRCs of the QZS isolator and ELS with different offset displacements and excitation amplitudes for the force excitation. "Red line" the disturbed isolator with $\widehat{u}_{0}=0.04$; "blue line" the disturbed isolator with $\widehat{u}_{0}=0.07$; "black line" the ideal isolator; "magenta line" ELS; "green dotted line" unstable solutions; "o" peak amplitude of response. 
with the increasing excitation amplitude. The FRCs of the ELS are shifted upwards in the whole frequency region and the peak amplitudes always occur at $\Omega=1$ as the excitation amplitude increases.

The stiffness characteristics of the QZS isolator are also influenced by the excitation amplitude. The QZS isolator exhibits linear stiffness when the excitation amplitude is small. As the excitation amplitude increases, the ideal isolator always exhibits the hardening stiffness feature. But for the disturbed isolator, as the excitation amplitude increases, it owns the softening stiffness and then enters a region with softening stiffness at first and the hardening stiffness later on. If the excitation amplitude is large enough, the disturbed isolator only exhibits the hardening stiffness.

Another important conclusion can be drawn when the excitation amplitude of the QZS isolator is same as that of the ELS. At low frequencies, the amplitude of the harmonic term $A_{1}$ for the QZS isolator is larger than that for the ELS, while the amplitude of the harmonic term $A_{1}$ for the ideal isolator is larger than that for the disturbed isolator. In the region around the resonance frequency of the ELS, the amplitude of the harmonic term $A_{1}$ for the QZS isolator changes to be smaller than that for the ELS, while the amplitude of the harmonic term $A_{1}$ for the ideal isolator changes to be smaller than that for the disturbed isolator; at high frequencies, the amplitudes of the harmonic term $A_{1}$ for the QZS isolator and ELS approach to be the same level.

Figure 12 shows the FRCs of the QZS isolator and the ELS for the displacement excitation. The vertical coordinate is the relative displacement. Different from the FRCs for the force excitation, the peak amplitude of the harmonic term $A_{1}$ for the QZS isolator will show the unbounded value and that for the disturbed isolator cannot be smaller than that for the ideal isolator. The amplitude of the harmonic term $A_{1}$ for the QZS isolator and ELS will approach to the excitation amplitude with the increasing excitation frequency. The amplitude of the harmonic term $A_{1}$ for the ideal isolator reaches the excitation amplitude at lower frequency than that for the disturbed isolator, while the disturbed isolator reaches the excitation amplitude at lower frequency than that for the ELS.

\subsection{Effects of the Offset Displacement and Excitation Ampli-} tude on the Transmissibilities. The key indexes to evaluate the performance of an isolator are the force transmissibility for the force excitation and the absolute displacement transmissibility for the displacement excitation. Based on (21) and according to the investigation of Ravindra and Mallik [22], one can draw a conclusion that the absolute displacement transmissibility cannot perform satisfactorily with the isolation performance of the QZS isolator at high frequencies for the displacement excitation. Therefore, the absolute acceleration transmissibility for the displacement excitation is introduced to be another index to evaluate the isolation performance.

5.2.1. Force Transmissibility. The force transmissibility is defined as the ratio between the amplitude of the nondimensional dynamic force transmitted to the base and that of the nondimensional excitation force. It can be expressed by

$$
T_{f}=\frac{\widehat{F}_{t}}{\rho}
$$

where $\widehat{F}_{t}=\sqrt{\widehat{F}_{t e}^{2}+\widehat{F}_{t d}^{2}}, \widehat{F}_{t e}$ is the nondimensional elastic force and $\widehat{F}_{t d}$ is the nondimensional damping force.

For the disturbed isolator, the solution of (20) can be assumed to be

$$
\widehat{v}=A_{0}^{\prime}+A_{1} \cos (\Omega \tau+\varphi),
$$

where the bias term $A_{0}^{\prime}=A_{0} \mp \widehat{u}_{0}$.

According to (20), the nondimensional elastic force is

$$
\widehat{F}_{t e}=\alpha_{1} \widehat{v} \pm \alpha_{2} \widehat{v}^{2}+\chi \widehat{v}^{3} .
$$

Substituting (40) into (41), the nondimensional elastic force can be obtained as

$$
\widehat{F}_{t e}=\widehat{F}_{t 0}+\widehat{F}_{t 1} \cos (\Omega \tau+\varphi),
$$

where $\widehat{F}_{t 0}=\alpha_{1} A_{0}^{\prime} \pm \alpha_{2} A_{0}^{\prime 2} \pm\left(\alpha_{2} A_{1}^{2} / 2\right)+\chi A_{0}^{\prime 3}+(3 / 2) \chi A_{0}^{\prime} A_{1}^{2}$ and $\widehat{F}_{t 1}=\alpha_{1} A_{1} \pm 2 \alpha_{2} A_{0}^{\prime} A_{1}+(3 / 4) \chi A_{1}^{3}+3 \chi A_{0}^{\prime 2} A_{1}$.

By considering the dynamic force only, one can get the force transmissibility of the disturbed isolator:

$$
T_{f}=\frac{\sqrt{\widehat{F}_{t 1}^{2}+\left(2 \xi \Omega A_{1}\right)^{2}}}{\beta} .
$$

For the ideal isolator and ELS, their force transmissibilities can be expressed separately by [5]

$$
\begin{aligned}
& T_{f}=\frac{\sqrt{\left(\chi A_{1}^{3}\right)^{2}+\left(2 \xi \Omega A_{1}\right)^{2}}}{\beta}, \\
& T_{f l}=\sqrt{\frac{1+(2 \xi \Omega)^{2}}{\left(1-\Omega^{2}\right)^{2}+(2 \xi \Omega)^{2}}} .
\end{aligned}
$$

5.2.2. Absolute Displacement and Acceleration Transmissibilities. The absolute displacement transmissibility is defined as the ratio between the amplitude of the nondimensional absolute displacement of the mass and nondimensional excitation displacement. It is given by

$$
T_{d}=\frac{|v|}{\rho}
$$

For the disturbed isolator, the nondimensional absolute displacement of the mass can be expressed by

$$
\widehat{v}=\widehat{e} \mp \widehat{u}_{0}+\widehat{z}=A_{0} \mp \widehat{u}_{0}+A_{1} \cos (\Omega \tau+\varphi)+\rho \cos (\Omega \tau) \text {. }
$$

Then its absolute displacement transmissibility can be derived as

$$
T_{d}=\frac{\left|A_{0} \mp \widehat{u}_{0}\right|+\sqrt{A_{1}^{2}+\rho^{2}+2 A_{1} \rho \cos (\varphi)}}{\rho} .
$$



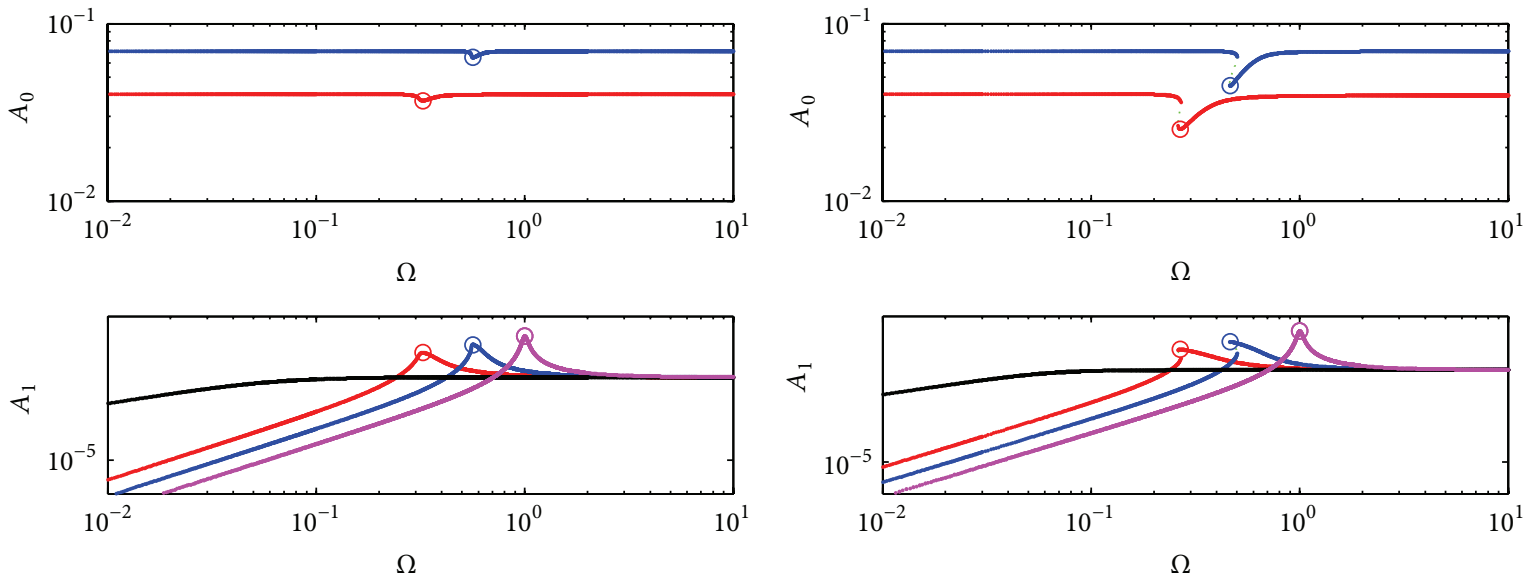

(a) $\rho=3 \times 10^{-3}$

(b) $\rho=8 \times 10^{-3}$
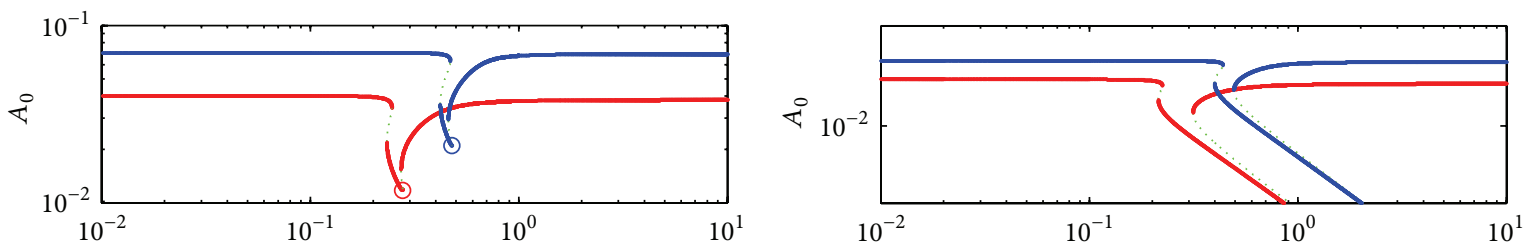

$\Omega$

$\Omega$

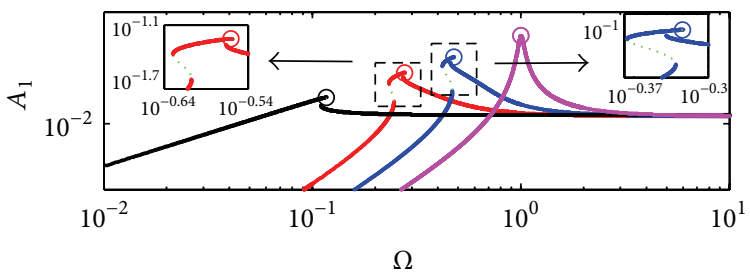

(c) $\rho=1.3 \times 10^{-2}$

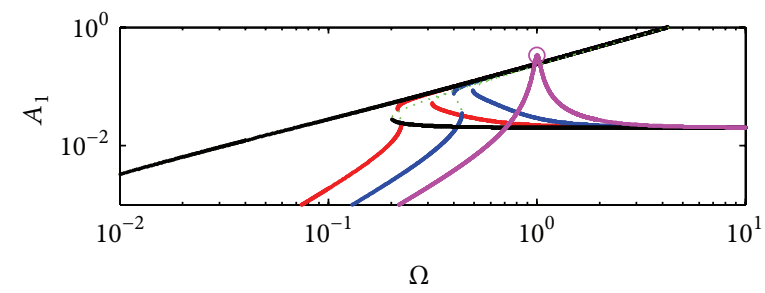

(d) $\rho=2 \times 10^{-2}$

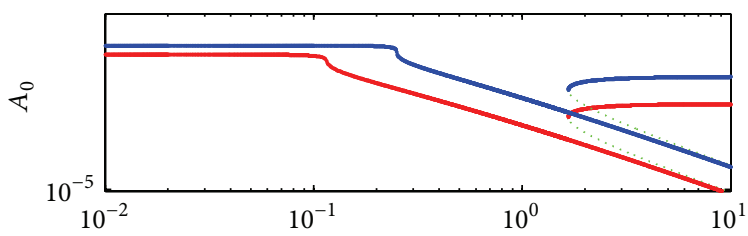

$\Omega$

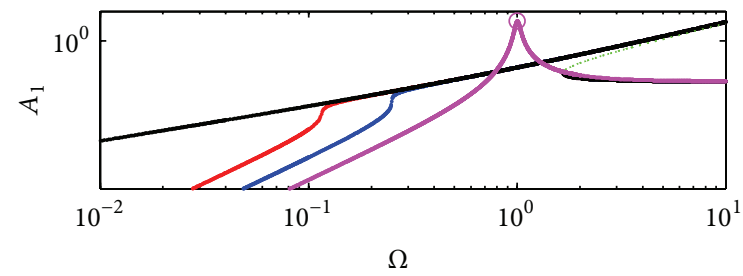

(e) $\rho=1.5 \times 10^{-1}$

FIGURE 12: FRCs of the QZS isolator and ELS with different offset displacements and excitation amplitudes for the displacement excitation. "Red line" the disturbed isolator with $\widehat{u}_{0}=0.04$; "blue line" the disturbed isolator with $\widehat{u}_{0}=0.07$; "black line" the ideal system; "magenta line" ELS; "green dotted line" unstable solutions; "o" peak amplitude of response. 
Its absolute acceleration transmissibility can be obtained as

$$
\begin{aligned}
T_{a c} & =\frac{|\ddot{\delta}|}{|\ddot{z}|}=\frac{\left|\widehat{\delta}^{\prime \prime}\right|}{\left|\widehat{z}^{\prime \prime}\right|}=\frac{\left|\widehat{v}^{\prime \prime}+\widehat{z}^{\prime \prime}\right|}{\left|\widehat{z}^{\prime \prime}\right|} \\
& =\frac{\left|\widehat{e}^{\prime \prime}+\widehat{z}^{\prime \prime}\right|}{\left|\widehat{z}^{\prime \prime}\right|}=\frac{\sqrt{A_{1}^{2}+\rho^{2}+2 A_{1} \rho \cos (\varphi)}}{\rho} .
\end{aligned}
$$

The absolute displacement and acceleration transmissibilities of the ideal isolator have the same expression as [5]

$$
T_{d}=T_{a c}=\frac{\sqrt{A_{1}^{2}+\rho^{2}+2 A_{1} \rho \cos (\varphi)}}{\rho},
$$

where $\cos (\varphi)$ can be obtained by setting $A_{0}=0$ in (23b). And the absolute displacement and acceleration transmissibilities of the ELS also have the same expression as (45).

Figure 13 presents the force transmissibility for the force excitation and Figure 14 presents the absolute displacement and acceleration transmissibilities for the displacement excitation. Note that all the transmissibility results are plotted in $\mathrm{dB}$, that is, $20 \log _{10} T$. By inspecting Figures 13 and 14 , one can conclude that the isolation performance of the QZS isolator will be better or worse than that of the ELS depending on the frequency range and excitation amplitude.

As shown in Figure 13, smaller offset displacement yields smaller peak amplitude and smaller resonance frequency of the force transmissibility when the excitation amplitude is fixed. Since the characteristics of the QZS isolator change with the increasing excitation amplitude, the peak amplitude of the force transmissibility for the ideal isolator is larger than that of the disturbed isolator with a small offset displacement. Different from that of the ELS independent of the excitation amplitude, the force transmissibility of the QZS isolator is affected obviously with the increasing excitation amplitude when the offset displacement is fixed. For the ideal isolator, larger excitation amplitude results in larger peak amplitude of the force transmissibility. However, it is worthy of note that the peak amplitude of the force transmissibility for the disturbed isolator decreases at first, increases later, and becomes larger than that of the ELS finally as the excitation amplitude increases.

When the excitation amplitude of the QZS isolator is same as that of the ELS, the force transmissibility of the QZS isolator is larger than that of the ELS, while the force transmissibility of the ideal isolator is larger than that of the disturbed isolator at low frequencies. In the region around the resonance frequency of the ELS, the force transmissibility of the QZS isolator changes to be smaller than that for the ELS, while the force transmissibility of the ideal isolator changes to be smaller than that of the disturbed isolator; at high frequencies, the force transmissibilities of the QZS isolator and the ELS approach to be the same level.

Unlike the force transmissibility, the peak amplitudes of the absolute displacement and acceleration transmissibilities for the disturbed isolator cannot be smaller than that for the ideal isolator as shown in Figure 14. And the QZS isolator owns unbounded absolute displacement and acceleration transmissibilities if the excitation amplitude is relatively large. For the disturbed isolator, the absolute displacement transmissibility is always larger than the absolute displacement transmissibility, which is also confirmed by (48) and (49). At low frequencies and region around the resonance frequency of the ELS, the interactive feature of the absolute displacement and acceleration transmissibilities between the QZS isolator and ELS for same excitation amplitude are same as that of the force transmissibility. At high frequencies, the absolute acceleration transmissibilities of the QZS isolator and ELS approach to be the same level, while the absolute displacement transmissibility of the disturbed isolator is larger than that of the ideal isolator and ELS. It is also worth noting that the absolute displacement transmissibility of the disturbed isolator at high frequencies increases as the excitation amplitude increases.

5.3. Effects of the Damping Ratio on the Transmissibilities. In the analysis above, the damping ratio of the QZS isolator is always fixed as $\xi=0.03$. It is interesting to study the effects of the damping ratio on the transmissibilities of the disturbed isolator when the offset displacement and excitation amplitude are fixed. For the two types of excitations, the values of the offset displacement $\widehat{u}_{0}$ and excitation amplitude $\rho$ are chosen to be $\widehat{u}_{0}=0.07, \rho=2.7 \times 10^{-3} ; \widehat{u}_{0}=0.07, \rho=$ $1.3 \times 10^{-2}$ separately. There will be the occurrence of five steady-state values for the disturbed isolator in the two cases. The transmissibilities of the disturbed isolator, the ideal isolator, and their ELS with different damping ratio are plotted in Figure 15. It can be seen obviously that larger damping ratio can result in smaller peak amplitudes of the transmissibilities around the resonance frequency but worse isolation performance at high frequencies. For the disturbed isolator and ideal isolator, an increase in the damping ratio yields a decrease in the maximum number of the steady-state values. And the larger damping ratio the fewer the jump points, which means that the damper can be used to avoid the occurrence of the jumps for the nonlinear isolator. For the ideal isolator, peak amplitudes of the transmissibilities will not occur if the damping ratio is large enough, which means that the ideal isolator can isolate the vibration at a lower frequency than its ELS. For the disturbed isolator, it is worthy of note that the resonance frequency decreases at first and increases later on with the increasing damping ratio.

\section{Conclusions}

In this paper, we introduce the theoretical design and characteristics analysis of a novel QZS isolator. The QZS isolator is developed by adding a disk spring with negative stiffness to a vertical linear spring with positive stiffness. The disk spring can offer great support capacity with small deflection and the QZS isolator is suitable for being used in the occasion with space limitation for isolators.

The static characteristics of the disk spring with variable thickness are investigated and compared to that of the 


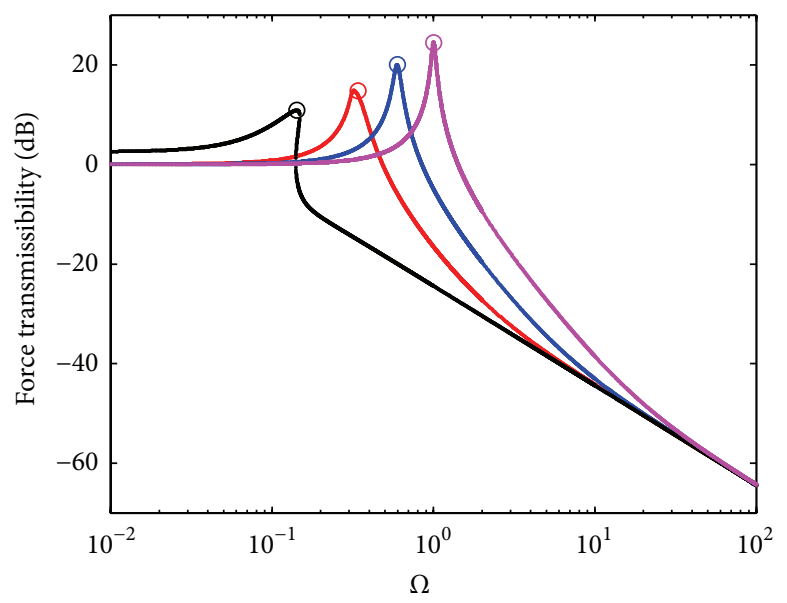

(a) $\rho=3 \times 10^{-4}$

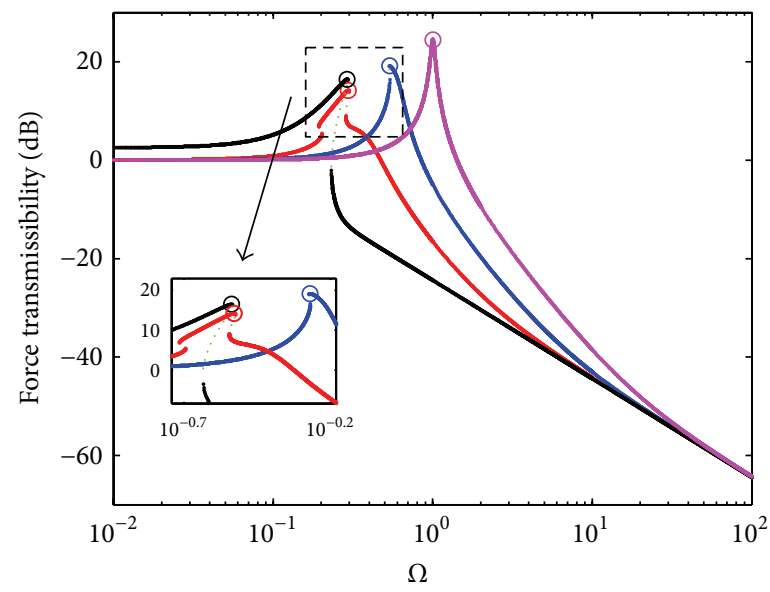

(c) $\rho=1.2 \times 10^{-3}$

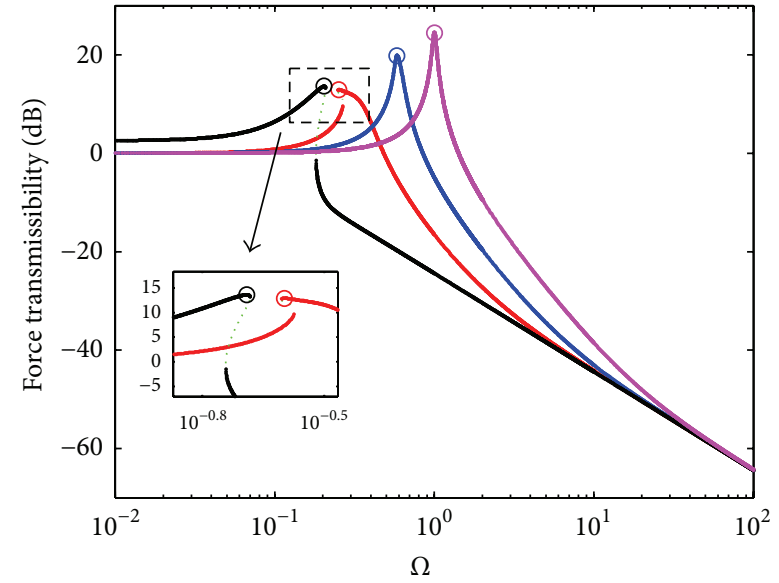

(b) $\rho=6 \times 10^{-4}$

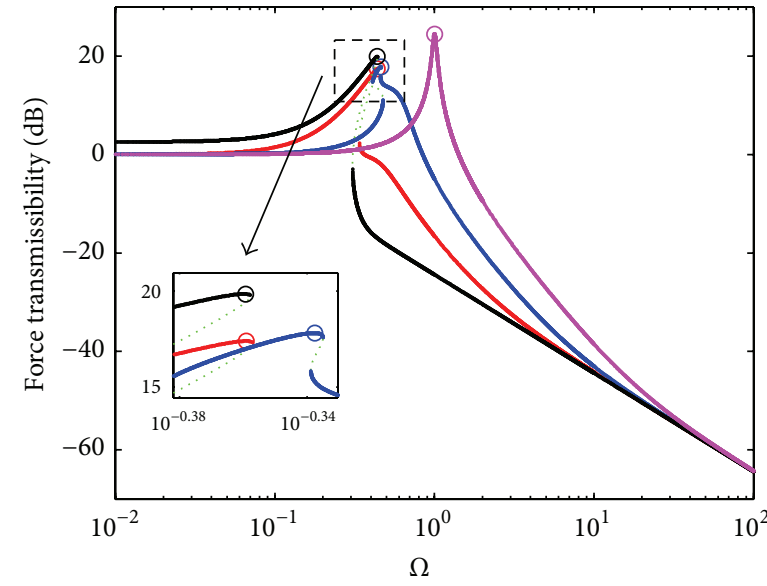

(d) $\rho=2.7 \times 10^{-3}$

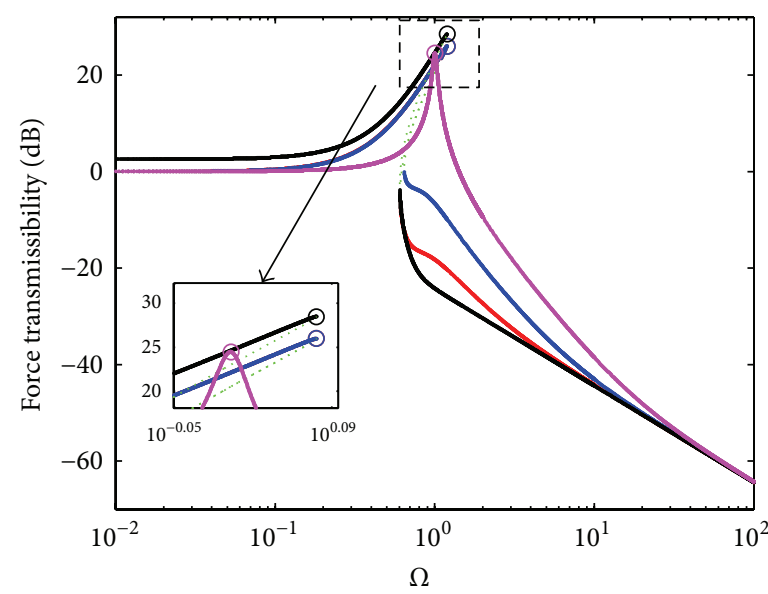

(e) $\rho=2 \times 10^{-2}$

FIGURE 13: Force transmissibilities of QZS isolator and ELS with different offset displacements and excitation amplitudes for the force excitation. "Red line" the disturbed isolator with $\widehat{u}_{0}=0.04$; "blue line" the disturbed isolator with $\widehat{u}_{0}=0.07$; "black line" the ideal isolator; "magenta line" ELS; "green dotted line" unstable solutions; "o" peak amplitude of transmissibility. 


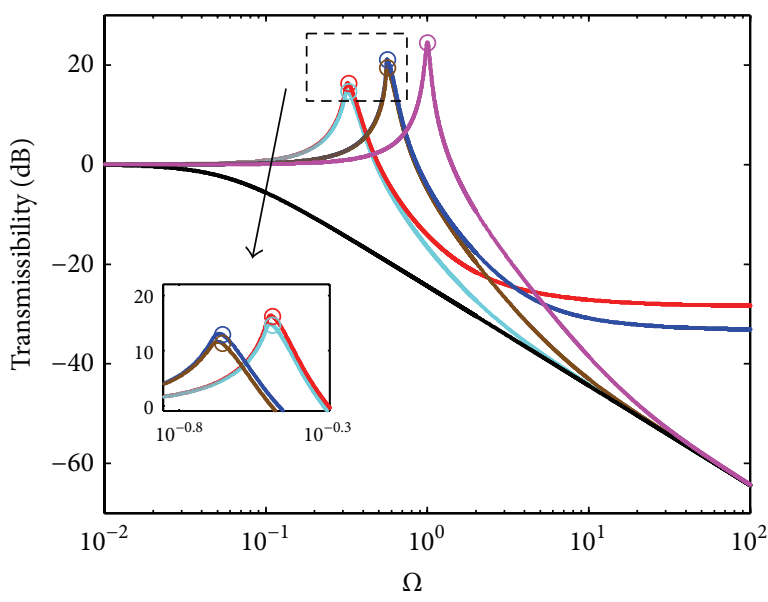

(a) $\rho=3 \times 10^{-3}$

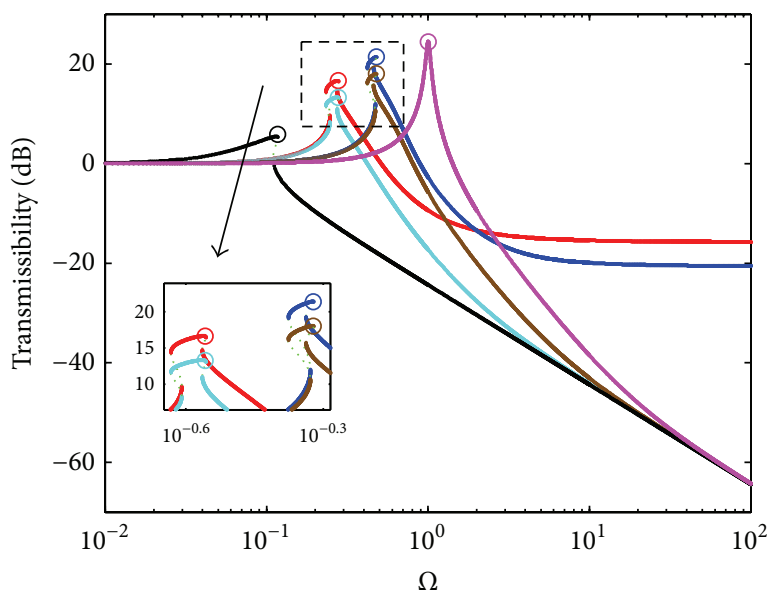

(c) $\rho=1.3 \times 10^{-2}$

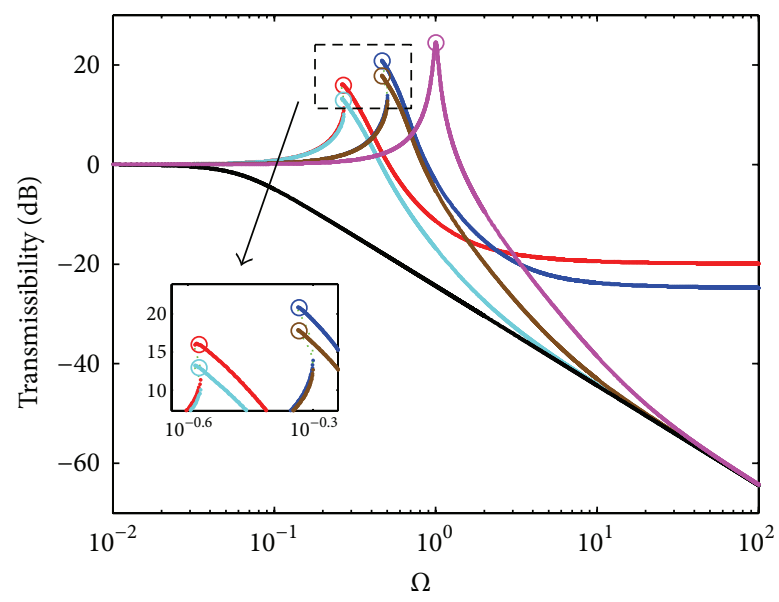

(b) $\rho=8 \times 10^{-3}$

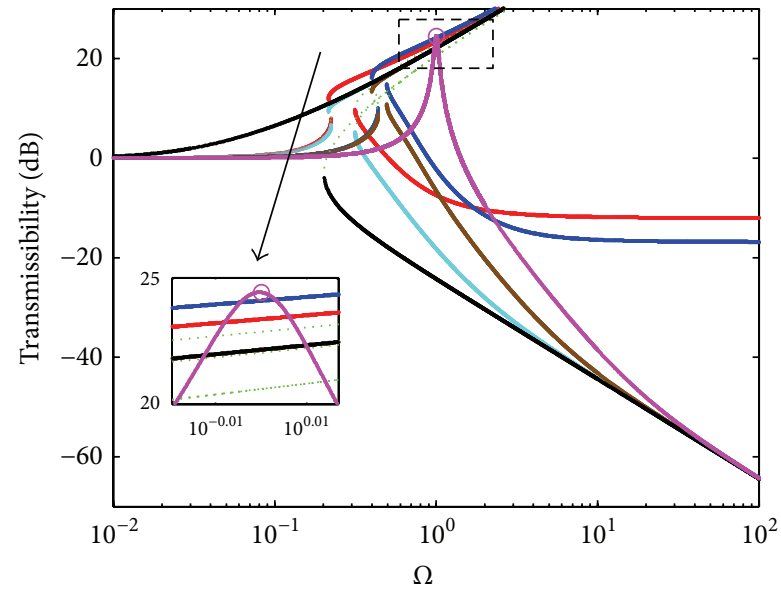

(d) $\rho=2 \times 10^{-2}$

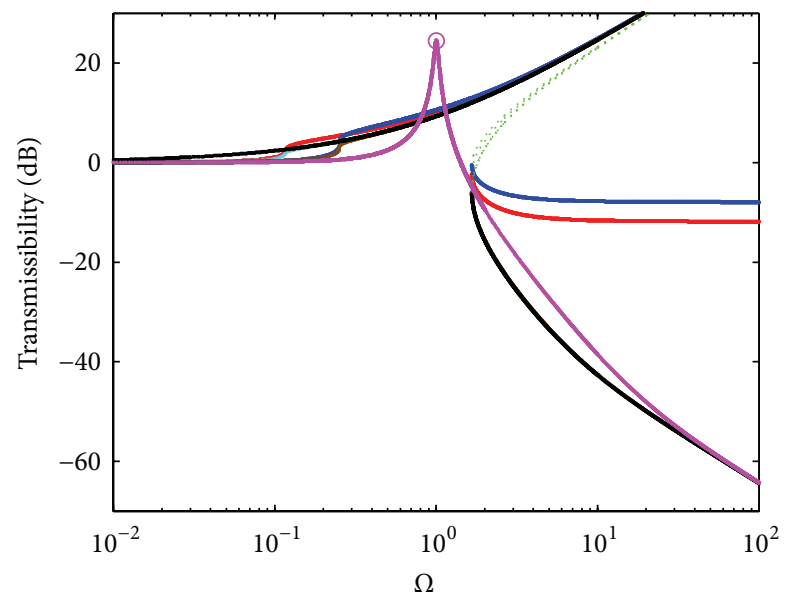

(e) $\rho=1.5 \times 10^{-1}$

FIGURE 14: Absolute displacement and acceleration transmissibilities of the QZS isolator and ELS with different offset displacements and excitation amplitudes for the displacement excitation. "Red line" absolute displacement transmissibility of the disturbed isolator with $\widehat{u}_{0}=0.04$; "cyan line" absolute acceleration transmissibility of the disturbed isolator with $\widehat{u}_{0}=0.04$; "blue line" absolute displacement transmissibility of the disturbed isolator with $\widehat{u}_{0}=0.07$; "brown line" absolute acceleration transmissibility of the disturbed isolator with $\widehat{u}_{0}=0.07$; "black line" the ideal isolator; "magenta line" ELS; "green dotted line" unstable solutions; "o" peak amplitude of transmissibility. 


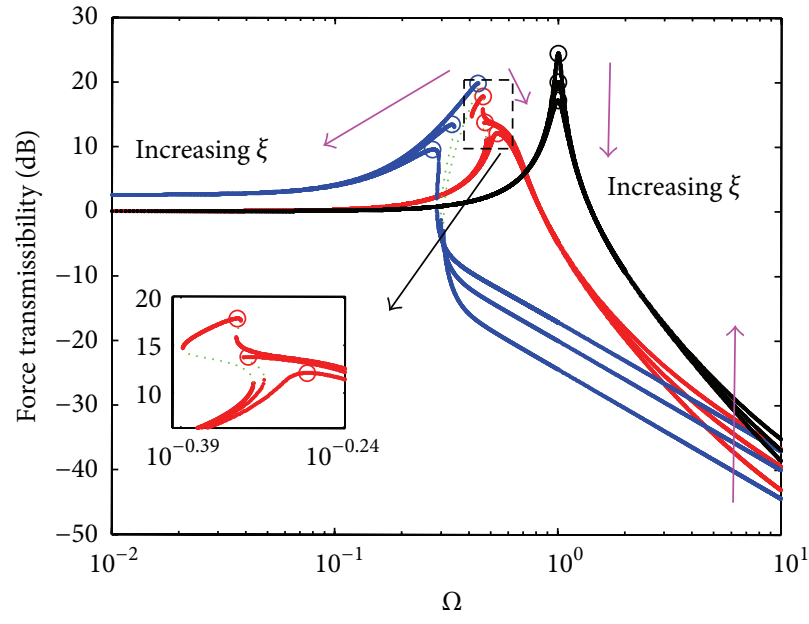

(a) Force transmissibility

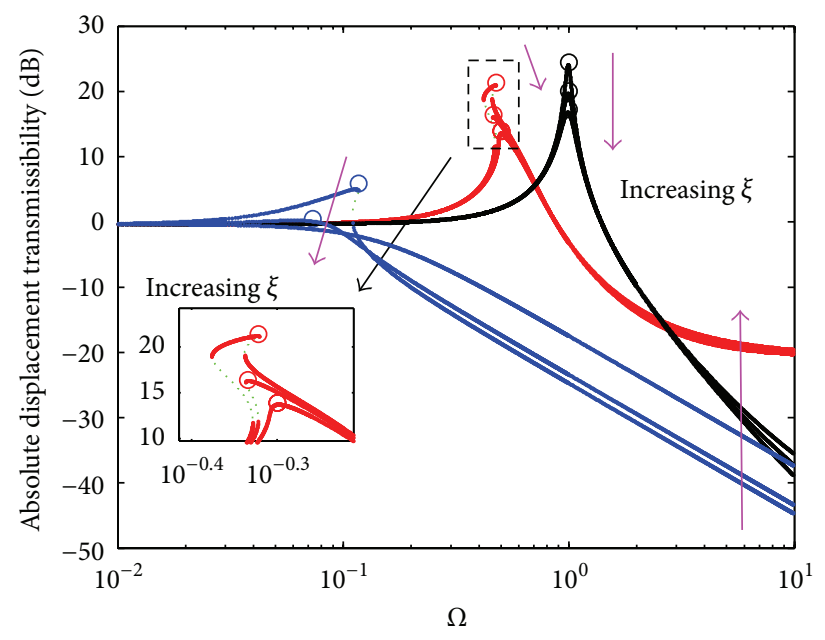

(b) Absolute displacement transmissibility

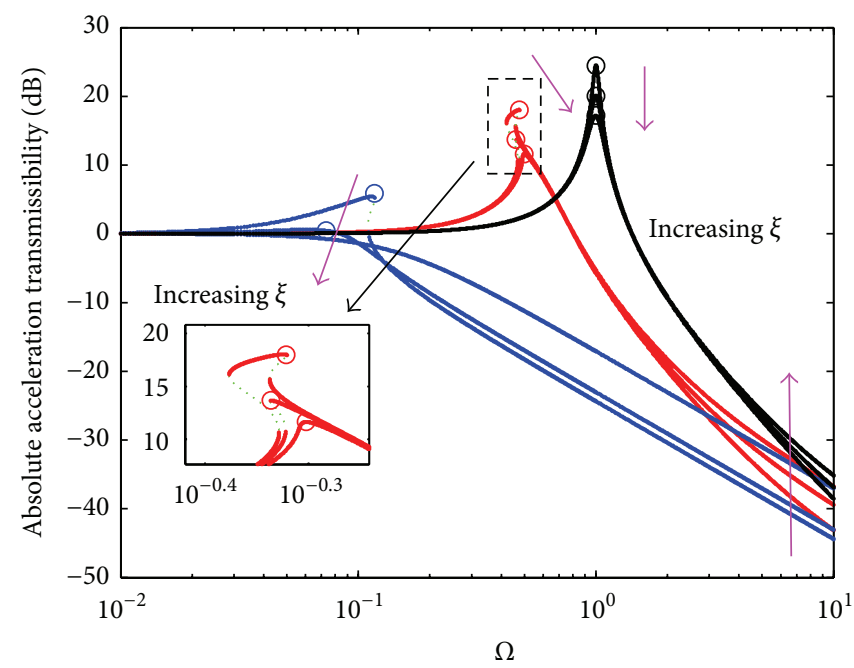

(c) Absolute acceleration transmissibility

FIGURE 15: Transmissibilities of the QZS isolator and ELS with different damping ratios $(\xi=0.03 ; \xi=0.05 ; \xi=0.07)$. "Red line" the disturbed isolator; "blue line" the ideal isolator; "black line" ELS; "green dotted line" unstable solutions; "o" peak amplitude of transmissibility; "magenta arrow" increasing damping ratio.

constant thickness disk spring. The relationship of the configurative parameters and defined stiffness ratio is obtained for achieving the zero stiffness at the static equilibrium position. Combinations of the configurative parameters are optimized for a wide displacement range around the static equilibrium position with a low dynamic stiffness and the stiffness changing slightly.

The overloaded and underloaded conditions, which result in an offset displacement of the static equilibrium position from the zero stiffness position, are taken into consideration. Considering only the primary resonance response, the steady-state solutions for the force and displacement excitations are got by employing the HBM and confirmed by the results of numerical simulation. Maximum number of the steady-state values as a function of the offset displacement and excitation amplitude are discussed, respectively, for the two types of excitations. The phenomena of multiple jumps are illustrated and the stability is studied by applying Floquet theory.

The frequency response curves of the ideal and disturbed isolator for the two types of excitations have been plotted with different combinations of the offset displacement and excitation amplitude. The results indicate that the disturbed isolator can exhibit linear, softening, and mixed softeninghardening and hardening stiffness as the excitation amplitude increases. The unbounded response can occur for the displacement excitation. And decreasing the offset displacement and excitation amplitude can expand the frequency region of isolation for the QZS isolator.

The isolation performance of the QZS isolator is evaluated by introducing the force transmissibility, the absolute displacement, and acceleration transmissibility and compared with the ELS for the two types of excitations. The effects of the damping ratio on the transmissibilities are discussed for the 
disturbed isolator. The conclusion can be summarized that if the load differs less with its supporting capability, the excitation amplitude is not too large and the damper is large, and the proposed QZS isolator possesses better isolation performance in low frequency range compared with the ELS. Increasing the damper appropriately is also a feasible way to avoid the occurrence of the jumps for the QZS isolator.

\section{Nomenclature}

a: External radius of the disk spring

$A_{0}$ : Bias term of the steady-state solution

$A_{1}$ : Amplitude of harmonic term of the steady-state solution

$A_{0 p}^{f}$ : Peak amplitude of $A_{0}$ for the force excitation

$A_{0 p}^{d}$ : Peak amplitude of $A_{0}$ for the displacement excitation

$A_{1 p}^{f}$ : Peak amplitude of $A_{1}$ for the force excitation

$b$ : $\quad$ Internal radius of the disk spring

c: Damping coefficient

C: $\quad$ Constant term of the response under parametric excitations

$d$ : $\quad$ Range of displacement from the static equilibrium position

$e$ : Transformed displacement of the mass under asymmetric excitation

$E$ : Young's modulus

$f(t)$ : Force excitation

$F$ : Amplitude of the force excitation

$F_{d}$ : Restoring force of the disk spring

$F_{d e}: \quad$ Restoring force of the disk spring when the disk spring in a horizontal line

$F_{r}$ : $\quad$ Restoring force of the QZS isolator

$F_{t}$ : Dynamic force transmitted to the base

$F_{t e}: \quad$ Elastic force

$F_{t d}:$ Damping force

$F_{t 0}$ : Constant term of $F_{t e}$

$F_{t 1}$ : Amplitude of harmonic term of $F_{t e}$

g: Acceleration of gravity

$h$ : $\quad$ Free height of the disk spring

$k$ : $\quad$ Stiffness of the QZS isolator

$k_{d}$ : $\quad$ Stiffness of the disk spring

$k_{d \text { min }}$ : The minimum negative value of $k_{d}$

$k_{v}$ : $\quad$ Stiffness of the vertical linear spring

$m$ : Weight of the mass

$n: \quad$ Integer

$P: \quad$ Axial force applied to the disk spring

$s: \quad$ Distance of neutral axis to center

$S: \quad$ Ultimate stress

$t_{a}: \quad$ Thickness at radius $a$

$t_{b}$ : Thickness at radius $b$

$t(p)$ : Thickness at radius $p$

$t_{s^{\prime}}: \quad$ Thickness at radius $s^{\prime}, s^{\prime}=(a+b) / 2$

$T_{a c}$ : Absolute acceleration transmissibility

$T_{d}$ : Absolute displacement transmissibility
$T_{f}: \quad$ Force transmissibility

$T_{f l}$ : Force transmissibility of the ELS

$u$ : Displacement response from the static equilibrium position

$u_{0}$ : Offset displacement

$u_{e}$ : Value of $u$ when the disk spring in a horizontal line

$v$ : Uniform of response of both excitation

$x$ : Deflection of the disk spring from initial position

$x_{e}: \quad$ Deflection $x$ when the disk spring in a horizontal line

$y$ : $\quad$ Relative displacement between the base and mass for displacement excitation

$z(t)$ : Displacement excitation

$Z$ : $\quad$ Amplitude of the displacement excitation

$\alpha$ : $\quad$ Constant term of asymmetric excitation

$\alpha_{1}$ : $\quad$ Parameter of linear term in dynamic equation

$\alpha_{2}$ : $\quad$ Parameter of square term in dynamic equation

$\beta$ : $\quad$ Initial cone angle of disk spring

$\gamma$ : Uniform parameter of both excitations

$\delta$ : Displacement from the new equilibrium position for overload

$\varepsilon: \quad$ Small perturbation

$\xi: \quad$ Damping ratio

$\eta$ : $\quad$ Constant term of the restoring force

$\theta: \quad$ Phase of the response under parametric excitations

$\iota_{0}$ : Constant term of Hill's equation

$\iota_{1}$ : Amplitude of the first harmonic term of Hill's equation

$\iota_{2}$ : Amplitude of the second harmonic term of Hill's equation

$\kappa: \quad$ Nondimensional parameter which defines the form of the spring

$\lambda$ : $\quad$ Stiffness ratio between the disk spring and the vertical linear spring

$\lambda_{\mathrm{QZS}}$ : Stiffness ratio when the stiffness of the QZS isolator equals zero

$\mu$ : Poisson's ratio

$\rho$ : Uniform amplitude of both excitations

$\tau: \quad$ Nondimensional time

$\phi$ : $\quad$ Change of cone angle due to load $P$

$\chi$ : $\quad$ Parameter of cubic term in dynamic equation

$\omega: \quad$ Excitation frequency

$\omega_{n}$ : Natural frequency of the QZS isolator without disk spring

$\Omega: \quad$ Frequency ratio $\omega / \omega_{n}$

$\Omega_{p}^{f}$ : Frequency corresponding to the peak response for force excitation

$\Omega_{p}^{d}$ : Frequency corresponding to the peak response for displacement excitation

$\varphi: \quad$ Phase of the response of both excitations

$\psi$ : Characteristic Floquet exponent. 


\section{Superscripts}

$\because$ Time derivative

: Nondimensional quantity

': Nondimensional time derivative.

\section{Conflict of Interests}

The authors declare that there is no conflict of interests regarding the publication of this paper.

\section{Acknowledgment}

The research was supported by the National Science and Technology Major Project of China (NSTMPS) under Grant no. 2012ZX10004801-002-013.

\section{References}

[1] J. Winterwood, High performance vibration isolation for gravitational wave detection [Ph.D. thesis], University of Western Australia, 2001.

[2] T. D. Le and K. K. Ahn, "A vibration isolation system in low frequency excitation region using negative stiffness structure for vehicle seat," Journal of Sound and Vibration, vol. 330, no. 26, pp. 6311-6335, 2011.

[3] C. M. Harris and A. G. Piersol, Shock and Vibrations Handbook, McGraw-Hill, New York, NY, USA, 2002.

[4] E. I. Rivin, Passive Vibration Isolation, ASME Press, New York, NY, USA, 2001.

[5] A. Carrella, M. J. Brennan, and T. P. Waters, "Static analysis of a passive vibration isolator with quasi-zero-stiffness characteristic," Journal of Sound and Vibration, vol. 301, no. 3-5, pp. 678689, 2007.

[6] R. A. Ibrahim, "Recent advances in nonlinear passive vibration isolators," Journal of Sound and Vibration, vol. 314, no. 3-5, pp. 371-452, 2008.

[7] P. Alabuzhev, A. Gritchin, L. Kim, G. Migirenko, V. Chon, and P. Stepanov, Vibration Protecting and Measuring Systems with Quasi-Zero Stiffness, Hemisphere, New York, NY, USA, 1989.

[8] X. Peng, D. Li, and S. Chen, "Quasi-zero stiffness vibration isolators and design for their elastic characteristics," Journal of Vibration, Measurement and Diagnosis, vol. 17, pp. 44-46, 1997.

[9] D. L. Platus, "Negative-stiffness-mechanism vibration isolation systems," in Optomechanical Engineering and Vibration Control, Proceedings of the SPIE, pp. 98-105, July 1999.

[10] J. Z. Zhang, D. Li, S. Dong, and M. J. Chen, "An ultra-low frequency parallel connection nonlinear isolator for precision instruments," Key Engineering Materials, vol. 257-258, pp. 231236, 2004.

[11] A. Carrella, Passive vibration isolators with high-static-lowdynamic-stiffness [Ph.D. thesis], ISVR, University of Southampton, 2008.

[12] I. Kovacic, M. J. Brennan, and T. P. Waters, "A study of a nonlinear vibration isolator with a quasi-zero stiffness characteristic," Journal of Sound and Vibration, vol. 315, no. 3, pp. 700-711, 2008.

[13] T. D. Le and K. K. Ahn, "Experimental investigation of a vibration isolation system using negative stiffness structure," International Journal of Mechanical Sciences, vol. 70, pp. 99-112, 2013.
[14] X. T. Liu, X. C. Huang, and H. X. Hua, "On the characteristics of a quasi-zero stiffness isolator using Euler buckled beam as negative stiffness corrector," Journal of Sound and Vibration, vol. 332, no. 14, pp. 3359-3376, 2013.

[15] A. Carrella, M. J. Brennan, T. P. Waters, and K. Shin, "On the design of a high-static-low-dynamic stiffness isolator using linear mechanical springs and magnets," Journal of Sound and Vibration, vol. 315, no. 3, pp. 712-720, 2008.

[16] W. S. Robertson, M. R. F. Kidner, B. S. Cazzdato, and A. C. Zander, "Theoretical design parameters for a quasi-zero stiffness magnetic spring for vibration isolation," Journal of Sound and Vibration, vol. 326, pp. 88-103, 2009.

[17] N. Zhou and K. Liu, "A tunable high-static-low-dynamic stiffness vibration isolator," Journal of Sound and Vibration, vol. 329, no. 9, pp. 1254-1273, 2010.

[18] D. L. Xu, Q. P. Yu, J. X. Zhou, and S. R. Bishop, "Theoretical and experimental analyses of a nonlinear magnetic vibration isolator with quasi-zero-stiffness characteristic," Journal of Sound and Vibration, vol. 332, no. 14, pp. 3377-3389, 2013.

[19] J. O. Almen and A. Laszlo, "The uniform section disc spring," Transactions of ASME, vol. 58, no. 4, pp. 765-770, 1973.

[20] G. la Rosa, M. Messina, and A. Risitano, "Stiffness of variable thickness Belleville springs," Journal of Mechanical Design, Transactions of the ASME, vol. 123, no. 2, pp. 294-299, 2001.

[21] Y. H. Zhang, H. H. Liu, and D. C. Wang, Spring Manual, China Machine Press, Beijing, China, 2008.

[22] B. Ravindra and A. K. Mallik, "Performance of non-linear vibration isolators under harmonic excitation," Journal of Sound and Vibration, vol. 170, no. 3, pp. 325-337, 1994.

[23] I. Kovacic, M. J. Brennan, and B. Lineton, "On the resonance response of an asymmetric Duffing oscillator," International Journal of Non-Linear Mechanics, vol. 43, no. 9, pp. 858-867, 2008.

[24] G. A. Korn and T. M. Korn, Mathematical Handbook for Scientists and Engineers, McGraw-Hill, New York, NY, USA, 1961.

[25] A. H. Nayfeh and D. T. Mook, Nonlinear Oscillations, Wiley, New York, NY, USA, 1979. 

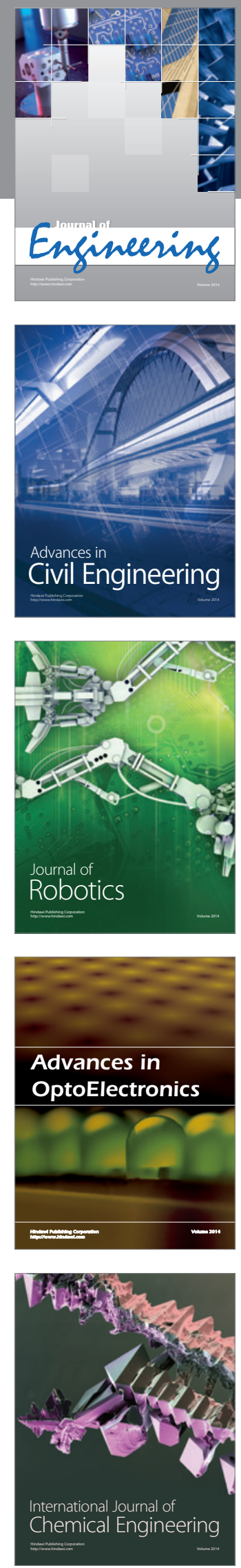

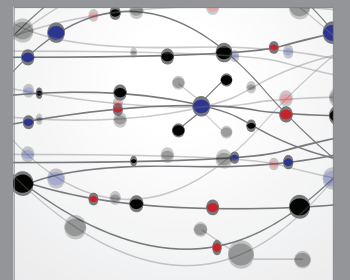

The Scientific World Journal
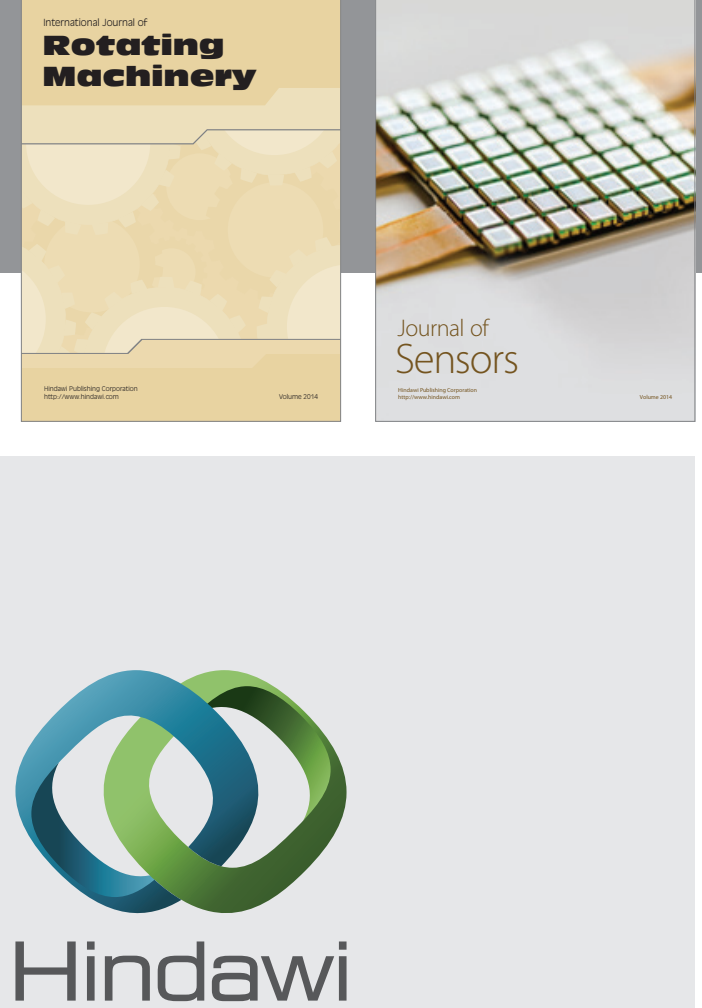

Submit your manuscripts at http://www.hindawi.com
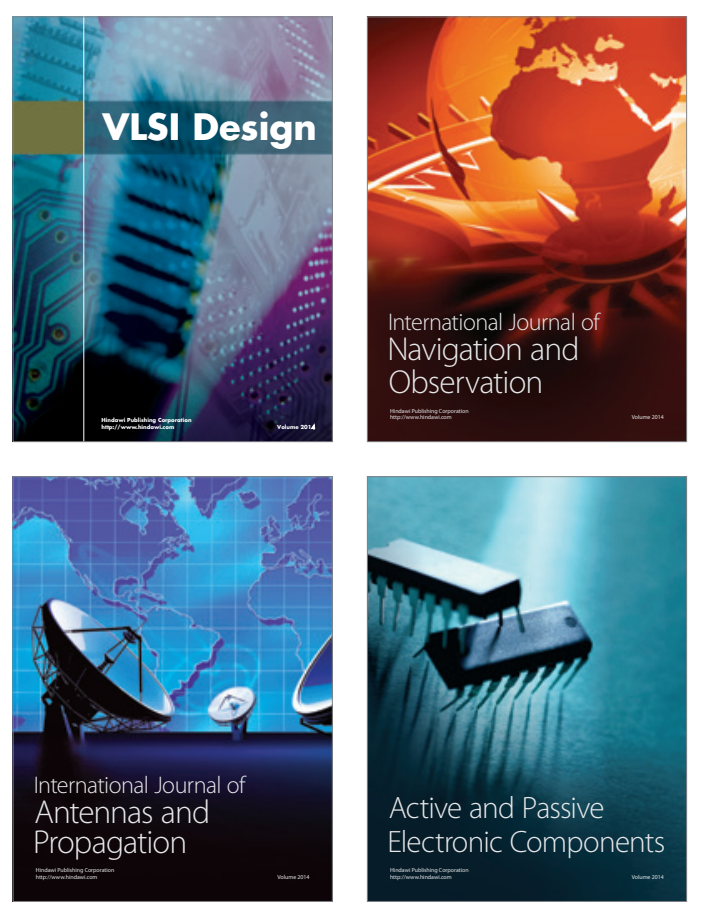
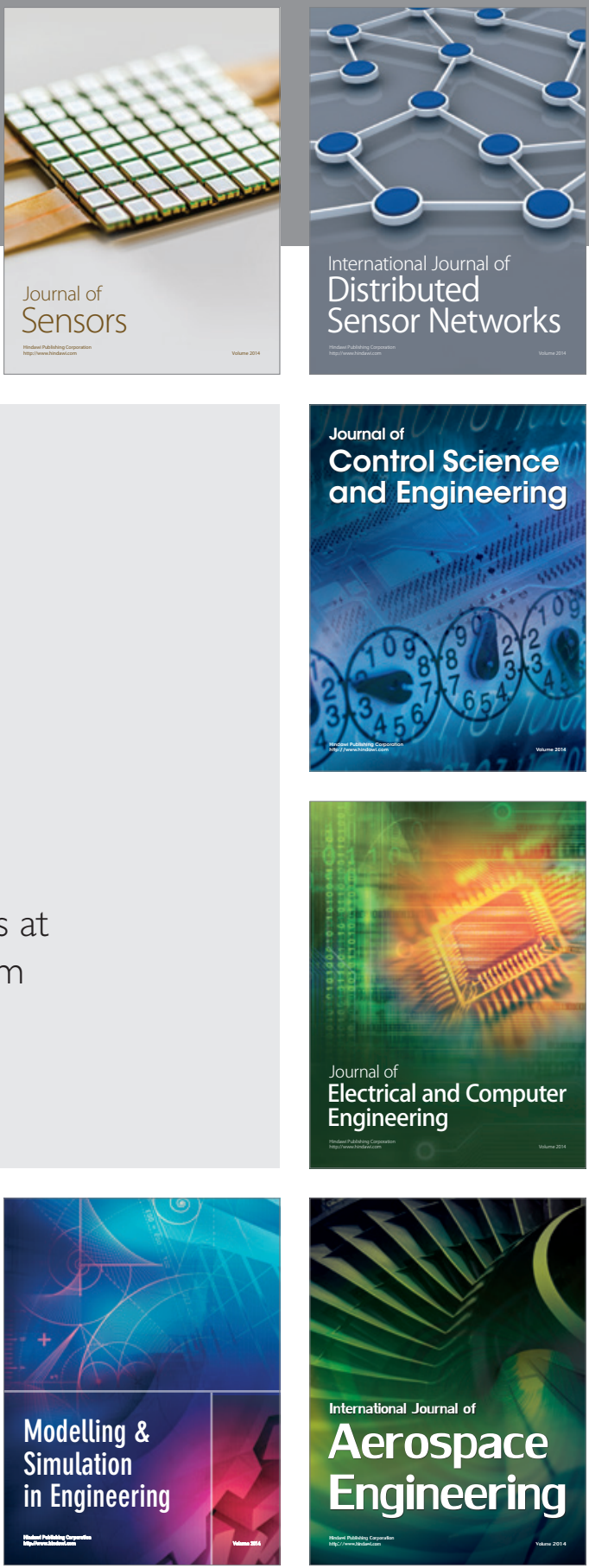

Journal of

Control Science

and Engineering
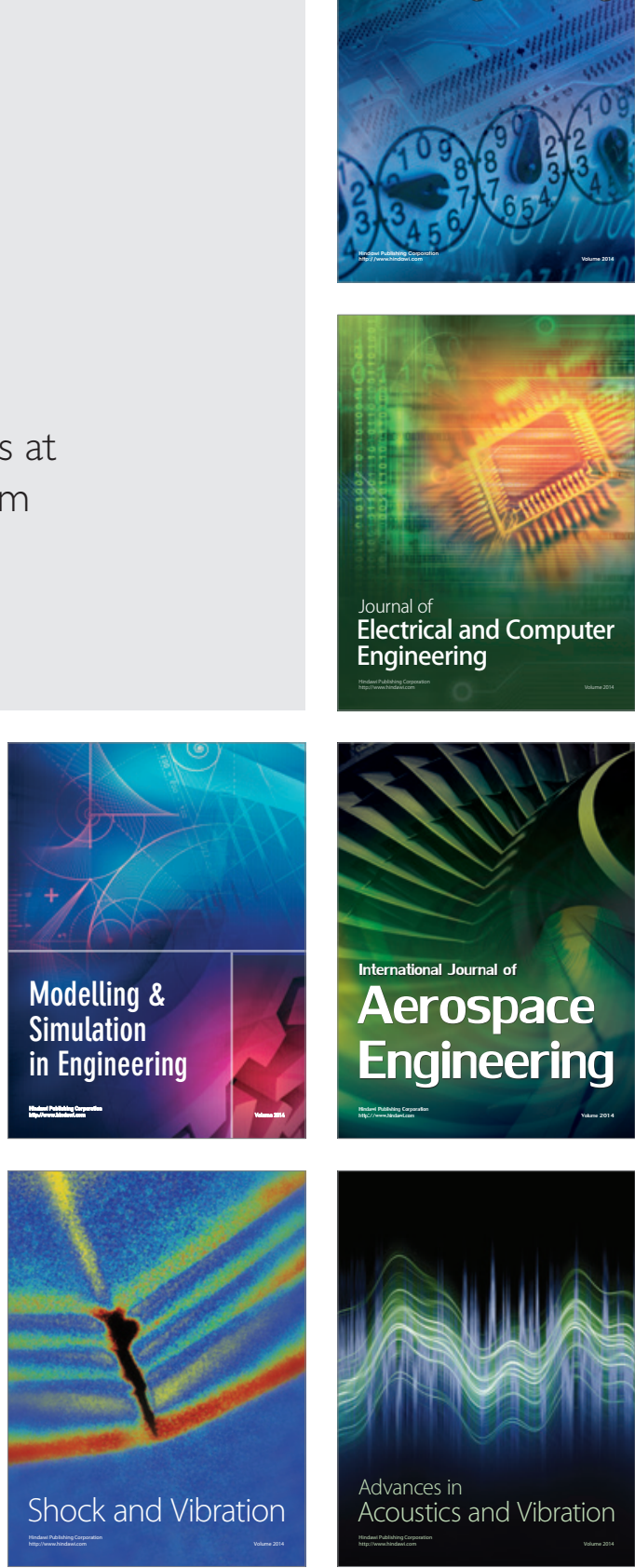